\title{
VISCOUS LIMITS TO PIECEWISE SMOOTH SOLUTIONS FOR THE NAVIER-STOKES EQUATIONS OF ONE-DIMENSIONAL COMPRESSIBLE VISCOUS HEAT-CONDUCTING FLUIDS*
}

\author{
SHIXIANG $\mathrm{MA}^{\dagger}$
}

\begin{abstract}
In this paper, we study the zero dissipation limit problem for the Navier-Stokes equations of one-dimensional compressible viscous heat-conducting fluids. We prove that if the solution of the inviscid Euler equations is piecewise smooth with finitely many noninteracting shocks satisfying the entropy condition, then there exist solutions to Navier-Stokes equations which converge to the inviscid solution away from shock discontinuities at a rate of $\varepsilon^{1}$ as the viscosity $\varepsilon$ tend to zero, provided that the heat-conducting coefficient $k=O(\varepsilon)$.
\end{abstract}

Key words. Compressible Navier-Stokes equations, compressible Euler equations, viscous limit, noninteracting shocks.

AMS subject classifications. 35Q30, 76N15

1. Introduction. The purpose of this paper is to study the asymptotic equivalence between the solutions of the compressible Navier-stokes equations and the compressible Euler equations. The one-dimensional Navier-stokes equations of compressible viscous heat-conducting fluids in Lagrangian coordinate are expressed as

$$
\left\{\begin{array}{l}
v_{t}-u_{x}=0 \\
u_{t}+p_{x}=\varepsilon\left(\frac{u_{x}}{v}\right)_{x}, \\
\left(e+\frac{u^{2}}{2}\right)_{t}+(p u)_{x}=\kappa\left(\frac{\theta_{x}}{v}\right)_{x}+\varepsilon\left(\frac{u u_{x}}{v}\right)_{x}, x \in R, t>0,
\end{array}\right.
$$

and the corresponding Euler system is of the form

$$
\left\{\begin{array}{l}
v_{t}-u_{x}=0 \\
u_{t}+p_{x}=0 \\
\left(e+\frac{u^{2}}{2}\right)_{t}+(p u)_{x}=0, \quad x \in R, \quad t>0,
\end{array}\right.
$$

where $v, u, \theta, p$ and $e$ denote the specific volume, the velocity, the temperature, the pressure, and the internal energy, respectively, and $\varepsilon, \kappa$ are the viscosity and heatconductivity coefficients, respectively. And $x$ is the Lagrangian coordinate, so that $x=$ constant corresponds to a particle path. Here we study the ideal polytropic gas, so that the pressure $p$ and the internal energy $e$ are related with $v$ and $\theta$ by the following equations of state

$$
p \equiv p(v, \theta)=R \theta / v, \quad e \equiv e(\theta)=R \theta /(\gamma-1)+\text { constant },
$$

where $R>0$ is the gas constant and $\gamma \in(1,2]$ is the adiabatic exponent.

In the theory of compressible fluids, the basic physics issue motivating the mathematical problem is the asymptotic equivalence between the viscous flows and the associate inviscid flows in the limit of small viscosity. This problem is particularly

\footnotetext{
*Received October 29, 2008; accepted for publication November 3, 2008.

$\dagger$ Department of Mathematics and The Institute of Mathematical Sciences, The Chinese University of Hong Kong, Shatin, N.T., Hong Kong (sxma@math.cuhk.edu.hk). Supported by NSFC (No. 10501015) and Tian yuan Fund (No. 10426016).
} 
important and of great significance in many physics phenomena and their numerical computations in the presence of shock discontinuities. When the underlying inviscid flow is smooth, this problem can be solved by classical methods. However, in the presence of shock discontinuities, the solutions near shock discontinuities exhibit very singular behavior as the viscosity is small. The rigorous mathematical justification of this asymptotic equivalence poses challenging problems in many important cases. For the viscous conservation laws with positive definite viscosity matrix, Goodman \& Xin [2], Yu [11], and Bianchini \& Bressan [18] studied the convergence of the solutions for the viscous conservation laws to those for the associated hyperbolic systems. In [2], Goodman and Xin gave a very detailed description of the asymptotic behavior of solutions to the viscous systems as this viscosity tends to zero for the case when the solutions of the associated hyperbolic conservation laws contain a finite number of non-interacting shocks, via a method of matching asymptotics. For the general solutions with the initial data having small total variations, Bianchini and Bressan proved the convergence of the solutions for the viscous systems to those for the associated hyperbolic systems by establishing the uniform (independent of viscosity) total variation estimates. The above results are for the viscous conservation laws with positive definite viscosity matrix. However, the viscosity matrix of the compressible Navier-Stokes equations (1.1) is only semi-positive definite, and thus less dissipative. For this case, when the flow is isentropic, Hoff \& Liu [7] and Wang [15] studied the limit process from the solutions of the compressible Navier-Stokes equations to the single shock-wave solution of the corresponding compressible Euler system(so called p-system). In [7], Hoff and Liu investigated the case when the underlying inviscid flow is a single weak shock wave. They show that the solutions to the isentropic Navier-Stokes equations with shock data exist and converge to the inviscid shocks as the viscosity vanishes, uniformly away from the shocks. And then by smooth initial perturbation, Wang [15] obtains the convergence rates. In this paper, we consider the full compressible Navier-Stokes equations. Motivated by [2] and [15], we use the matched asymptotic expansion analysis and energy estimates to establish that the piecewise smooth solutions of (1.2), with finitely many noninteracting shocks satisfying the entropy condition, are strong limits of solutions of (1.1) as the viscosity and heat-conductivity coefficients $\varepsilon, \kappa$ tend to zero. We assume that for some constant $C>0$,

$$
\kappa=O(\varepsilon) \text { as } \varepsilon \rightarrow 0 \text { and } \kappa(\varepsilon) / \varepsilon \geq C>0 .
$$

Without loss of generality, we set $\kappa=\varepsilon$. From the kinetic theory, the viscosity and heat-conductivity should be in the same order, the assumption (1.4) is reasonable [10].

For simplicity of presentation, we only consider the case in which the piecewise smooth solution $(v, u, \theta)$ to $(1.2)$ is a single-shock solution. And we assume that

$$
0<\underline{v} \leq v(x, t) \leq \bar{v} \text {, and } 0<\underline{\theta} \leq \theta(x, t) \leq \bar{\theta},
$$

for some constants $\underline{v}, \bar{v}, \underline{\theta}$ and $\bar{\theta}$.

Definition 1.1. A function $(v(x, t), u(x, t), \theta(x, t))$ is called a single-shock solution of (1.2) up to time $T$ if

i) $(v(x, t), u(x, t), \theta(x, t))$ is a distributional solution of the hyperbolic system (1.2) in the region $R^{1} \times[0, T]$.

ii) There is a smooth curve, the shock, $x=s(t), 0 \leq t \leq T$, so that $(v(x, t)$, $u(x, t), \theta(x, t))$ is sufficiently smooth at any point $x \neq s(t)$. 
iii) The limits

$$
\begin{aligned}
& \partial_{x}^{l}(v, u, \theta)(s(t)-0, t)=\lim _{x \rightarrow s(t)-} \partial_{x}^{l}(v, u, \theta)(x, t), \\
& \partial_{x}^{l}(v, u, \theta)(s(t)+0, t)=\lim _{x \rightarrow s(t)+} \partial_{x}^{l}(v, u, \theta)(x, t),
\end{aligned}
$$

exist and are finite for $t \leq T$ and $l \geq 0$.

iv) The Lax geometrical entropy condition [13] is satisfied at $x=s(t)$, that is,

$\dot{s}<\lambda_{1}(u(s(t)-0, t))$ and $\lambda_{1}(u(s(t)+0, t))<\dot{s}<\lambda_{2}(u(s(t)+0, t))$ (1-shocks), (1.6)

or

$\lambda_{2}(u(s(t)-0, t))<\dot{s}<\lambda_{3}(u(s(t)-0, t))$ and $\lambda_{3}(u(s(t)+0, t))<\dot{s}$ (3-shocks), (1.7)

where $\dot{s}=\frac{d}{d t} s(t)$ and $\lambda_{1}=-(\gamma R \theta)^{\frac{1}{2}} / v, \lambda_{2}=0, \lambda_{3}=(\gamma R \theta)^{\frac{1}{2}} / v$ are characteristic speeds of the hyperbolic system (1.2) with (1.3).

The Lax's shock condition implies that $\dot{s}<0$ for 1 -shocks and $\dot{s}>0$ for 3 -shocks. Here we only consider the 3 -shocks. Our main results are as follows:

TheOREM 1.2. Let $n \geq 3$ be an integer. Suppose that $(v, u, \theta)$ is a single-shock solution of system (1.2) up to time $T>0$ with

$$
\sum_{1 \leq k \leq(2 n+3)} \int_{0}^{T} \int\left|\partial_{x}^{k}(v(x, t), u(x, t), \theta(x, t))\right|^{2} d x d t<\infty .
$$

Then, there exist constants $\mu_{0}$ and $\varepsilon_{0}>0$, such that if

$$
(\gamma-1) \sup _{0 \leq t \leq T}|v(s(t)+0, t)-v(s(t)-0, t)| \leq \mu_{0}
$$

for any $\epsilon \in\left(0, \epsilon_{0}\right]$ there is a smooth solution $\left(v^{\varepsilon}, u^{\varepsilon}, \theta^{\varepsilon}\right)$ to (1.1) with the same initial data as the approximate solution $\left(\bar{v}^{\varepsilon}, \bar{u}^{\varepsilon}, \overline{\theta^{\varepsilon}}\right)$, constructed by (2.31), which is a small perturbation to $(v, u, \theta)$ in $L^{\infty}\left([0, T] ; L^{2}(R)\right)$. Moreover, it holds that

$$
\left(v^{\varepsilon}, u^{\varepsilon}, \theta^{\varepsilon}\right)(x, t)=\left(\bar{v}^{\varepsilon}, \bar{u}^{\varepsilon}, \bar{\theta}^{\varepsilon}\right)(x, t)+O\left(\epsilon^{n-\frac{7}{4}}\right),(x, t) \in R \times[0, T]
$$

in $L^{\infty}\left([0, T] ; L^{2}(R) \cap L^{\infty}(R)\right)$ and for any given $\eta \in(0,1)$ that

$$
\sup _{0 \leq t \leq T} \int\left|\left(v^{\varepsilon}, u^{\varepsilon}, \theta^{\varepsilon}\right)(x, t)-(v, u, \theta)(x, t)\right|^{2} d x \leq C_{\eta} \epsilon^{\eta},
$$

and

$$
\sup _{0 \leq t \leq T,|x-s(t)| \geq \epsilon^{\eta}}\left|\left(v^{\varepsilon}, u^{\varepsilon}, \theta^{\varepsilon}\right)(x, t)-(v, u, \theta)(x, t)\right| \leq C_{\eta} \epsilon
$$

where $C_{\eta}$ is a positive constant depending only on $\eta$.

REMARK 1.3. i) For the 1-shock, by a similar way, we can obtain the same results.

ii) The convergence rate in (1.12) is optimal. 
iii) The condition (1.9) implies that when $\gamma$ is close to $1^{+}$, the shock strength can be large.

iv)To prove Theorem 1.2 and to overcome the difficulties induced by nonisentropy of the flow, we shall adapt and modify the arguments in [2, 15, 16]. That is, we will exploit the smoothing property induced by the parabolic parts in (1.1), make best use of the properties of the shock profile and the smallness condition (1.9), and finally carefully compute the terms with different signs to deduce delicate energy estimates and so obtain the theorem.

Notation. In this paper, we use $H^{l}(l \geq 1)$ to denote the usual Sobolev space with the norm $\|\cdot\|_{l}$ and $\|\cdot\|=\|\cdot\|_{0}$ denotes the usual $L_{2}-$ norm. We also use $O(1)$ to denote any positive bounded function which is independent of $\epsilon$. And we set

$$
\mu \equiv \sup _{0 \leq t \leq T}|v(s(t)+0, t)-v(s(t)-0, t)|
$$

2. Construction of the approximate solutions. In this section, we construct the approximate solutions $\left(\bar{v}^{\varepsilon}, \bar{u}^{\varepsilon}, \bar{\theta}^{\varepsilon}\right)$ through different scaling and asymptotic expansions in the regions near and away from the shock respectively, such that $\left(\bar{v}^{\varepsilon}, \bar{u}^{\varepsilon}, \overline{\theta^{\varepsilon}}\right)$ approximates the piecewise smooth inviscid solution $(v, u, \theta)$ away from the shock and has a sharp change near the shock.

2.1. Outer and inner expansions and the matching conditions. Let $h_{i}(x, t)=\left(v_{i}, u_{i}, \theta_{i}\right)^{t}(x, t), i=0,1,2, \ldots$ In the region away from the shock, $x=s(t)$, we approximate the solution of (1.1) by truncating the formal series

$$
h^{\varepsilon}(x, t) \sim h_{0}(x, t)+\varepsilon h_{1}(x, t)+\varepsilon^{2} h_{2}(x, t)+\cdots
$$

Substituting this into (1.1) and comparing the coefficients of powers of $\varepsilon$, we get, for $x \neq s(t)$, that

$$
\begin{aligned}
& O(1):\left\{\begin{array}{l}
v_{0 t}-u_{0 x}=0 \\
u_{0 t}+p\left(v_{0}, \theta_{0}\right)_{x}=0 \\
\left(e_{0}+\frac{u_{0}^{2}}{2}\right)_{t}+\left(p\left(v_{0}, \theta_{0}\right) u_{0}\right)_{x}=0
\end{array}\right. \\
& O(\varepsilon):\left\{\begin{array}{l}
v_{1 t}-u_{1 x}=0 \\
u_{1 t}+\left(p_{v}\left(v_{0}, \theta_{0}\right) v_{1}+p_{\theta}\left(v_{0}, \theta_{0}\right) \theta_{1}\right)_{x}=\left(\frac{u_{0 x}}{v_{0}}\right)_{x} \\
\left(e_{1}+u_{0} u_{1}\right)_{t}+\left(p\left(v_{0}, \theta_{0}\right) u_{1}+u_{0}\left(p_{v}\left(v_{0}, \theta_{0}\right) v_{1}+p_{\theta}\left(v_{0}, \theta_{0}\right) \theta_{1}\right)\right)_{x} \\
=\left(\frac{\theta_{0 x}}{v_{0}}\right)_{x}+\left(\frac{u_{0} u_{0 x}}{v_{0}}\right)_{x}
\end{array}\right. \\
& O\left(\varepsilon^{2}\right):\left\{\begin{array}{l}
v_{2 t}-u_{2 x}=0, \\
u_{2 t}+\left(p_{v}\left(v_{0}, \theta_{0}\right) v_{2}+p_{\theta}\left(v_{0}, \theta_{0}\right) \theta_{2}\right)_{x}=\left(\frac{u_{1 x}}{v_{0}}-\frac{u_{0 x} v_{1}}{v_{0}^{2}}\right)_{x}-f_{1}\left(v_{0}, \theta_{0} ; v_{1}, \theta_{1}\right)_{x}, \\
\left(e_{2}+u_{0} u_{2}\right)_{t}+\left(p\left(v_{0}, \theta_{0}\right) u_{2}+u_{0}\left(p_{v}\left(v_{0}, \theta_{0}\right) v_{2}+p_{\theta}\left(v_{0}, \theta_{0}\right) \theta_{2}\right)\right)_{x} \\
=\left(\frac{\theta_{1 x}}{v_{0}}-\frac{\theta_{0 x} v_{1}}{v_{0}^{2}}\right)_{x}+\left(\frac{u_{1} u_{0 x}}{v_{0}}+\frac{u_{0} u_{1 x}}{v_{0}}-\frac{u_{0} u_{0 x} v_{1}}{v_{0}^{2}}\right)_{x} \\
\quad-\frac{1}{2}\left(u_{1}^{2}\right)_{t}-f_{2}\left(v_{0}, u_{0}, \theta_{0} ; v_{1}, u_{1}, \theta_{1}\right)_{x},
\end{array}\right.
\end{aligned}
$$


and etc., where $e_{i}=e\left(\theta_{i}\right), i=0,1,2, \cdots$, and

$$
\begin{aligned}
f_{1}\left(v_{0}, \theta_{0} ; v_{1}, \theta_{1}\right)= & \frac{1}{2}\left\{p_{v v}\left(v_{0}, \theta_{0}\right) v_{1}^{2}+2 p_{v \theta}\left(v_{0}, \theta_{0}\right) v_{1} \theta_{1}\right\}, \\
f_{2}\left(v_{0}, u_{0}, \theta_{0} ; v_{1}, u_{1}, \theta_{1}\right)= & u_{1}\left(p_{v}\left(v_{0}, \theta_{0}\right) v_{1}+p_{\theta}\left(v_{0}, \theta_{0}\right) \theta_{1}\right) \\
& +\frac{1}{2} u_{0}\left\{p_{v v}\left(v_{0}, \theta_{0}\right) v_{1}^{2}+2 p_{v \theta}\left(v_{0}, \theta_{0}\right) v_{1} \theta_{1}\right\},
\end{aligned}
$$

and etc. The outer functions $h_{0}, h_{1}, \ldots$, are generally discontinuous at the shock, $x=s(t)$, but smooth up to the shock. The leading term, $h_{0}$, is the single shock solution of (1.2) which is given in Theorem 1.2.

Near the shock, $h^{\varepsilon}$ should be represented by an inner expansion:

$$
h^{\varepsilon}(x, t) \sim H_{0}(\xi, t)+\varepsilon H_{1}(\xi, t)+\varepsilon^{2} H_{2}(\xi, t)+\cdots
$$

where

$$
\xi=\frac{x-s(t)}{\varepsilon}+\delta(t, \varepsilon)
$$

and $\delta(t, \varepsilon)$ is a perturbation of the shock position to be determined later. We assume that $\delta(t, \varepsilon)$ has the form

$$
\delta(t, \varepsilon)=\delta_{0}(t)+\varepsilon \delta_{1}(t)+\varepsilon^{2} \delta_{2}(t)+\cdots
$$

Substitute (2.5)-(2.7) into (1.1) to obtain

$$
\begin{gathered}
O\left(\frac{1}{\varepsilon}\right):\left\{\begin{array}{l}
-\dot{s} V_{0 \xi}-U_{0 \xi}=0, \\
-\dot{s} U_{0 \xi}+p\left(V_{0}, \Theta_{0}\right)_{\xi}=\left(\frac{U_{0 \xi}}{V_{0}}\right)_{\xi}, \\
-\dot{s}\left(E_{0}+\frac{U_{0}^{2}}{2}\right)_{\xi}+\left(p\left(V_{0}, \Theta_{0}\right) U_{0}\right)_{\xi}=\left(\frac{\Theta_{0 \xi}}{V_{0}}\right)_{\xi}+\left(\frac{U_{0} U_{0 \xi}}{V_{0}}\right)_{\xi},
\end{array}\right. \\
O(1):\left\{\begin{array}{l}
-\dot{s} V_{1 \xi}-U_{1 \xi}=-\dot{\delta_{0}} V_{0 \xi}-V_{0 t}, \\
-\dot{s} U_{1 \xi}+\left(p_{v}\left(V_{0}, \Theta_{0}\right) V_{1}+p_{\theta}\left(V_{0}, \Theta_{0}\right) \Theta_{1}\right)_{\xi} \\
=\left(\frac{U_{1 \xi}}{V_{0}}-\frac{U_{0 \xi} V_{1}}{V_{0}^{2}}\right)_{\xi}-\dot{\delta_{0}} U_{0 \xi}-U_{0 t}, \\
-\dot{s}\left(E_{1}+U_{0} U_{1}\right)_{\xi}+\left\{p\left(V_{0}, \Theta_{0}\right) U_{1}+\left(p_{v}\left(V_{0}, \Theta_{0}\right) V_{1}+p_{\theta}\left(V_{0}, \Theta_{0}\right) \Theta_{1}\right) U_{0}\right\}_{\xi} \\
=\left(\frac{\Theta_{1 \xi}}{V_{0}}-\frac{\Theta_{0 \xi} V_{1}}{V_{0}^{2}}\right)_{\xi}+\left(\frac{U_{1} U_{0 \xi}}{V_{0}}+\frac{U_{0} U_{1 \xi}}{V_{0}}-\frac{U_{0} U_{0 \xi} V_{1}}{V_{0}^{2}}\right)_{\xi} \\
-\dot{\delta_{0}}\left(E_{0}+\frac{U_{0}^{2}}{2}\right)_{\xi}-\left(E_{0}+\frac{U_{0}^{2}}{2}\right)_{t},
\end{array}\right.
\end{gathered}
$$




$$
O(\varepsilon):\left\{\begin{aligned}
- & \dot{s} V_{2 \xi}-U_{2 \xi}=-\dot{\delta_{1}} V_{0 \xi}-\dot{\delta_{0}} V_{1 \xi}-V_{1 t} \\
- & \dot{s} U_{2 \xi}+\left(p_{v}\left(V_{0}, \Theta_{0}\right) V_{2}+p_{\theta}\left(V_{0}, \Theta_{0}\right) \Theta_{2}\right)_{\xi} \\
= & \left\{\frac{U_{2 \xi}}{V_{0}}-\frac{U_{0 \xi} V_{2}}{V_{0}^{2}}+\mathcal{B}_{1}\left(V_{0}, U_{0} ; V_{1}, U_{1}\right)\right\}_{\xi}-f_{1}\left(V_{0}, \Theta_{0} ; V_{1}, \Theta_{1}\right)_{\xi} \\
& -\dot{\dot{\delta}} U_{0 \xi}-\dot{\delta_{0}} U_{1 \xi}-U_{1 t} \\
- & \left\{\frac{\left.\Theta_{2 \xi}+U_{0} U_{2}\right)_{\xi}+\left\{p\left(V_{0}, \Theta_{0}\right) U_{2}+\left(p_{v}\left(V_{0}, \Theta_{0}\right) V_{2}+p_{\theta}\left(V_{0}, \Theta_{0}\right) \Theta_{2}\right) U_{0}\right\}_{\xi}}{V_{0}}+\mathcal{B}_{1}\left(V_{0}, \Theta_{0} ; V_{1}, \Theta_{1}\right)\right\}_{\xi} \\
& +\left\{\frac{U_{2} U_{0 \xi}}{V_{0}}+\frac{U_{0} U_{2 \xi}}{V_{0}}-\frac{U_{0} U_{0 \xi} V_{2}}{V_{0}^{2}}+\mathcal{B}_{2}\left(V_{0}, U_{0} ; V_{1}, U_{1}\right)\right\}_{\xi} \\
& -\dot{U_{1}^{2}}\left(\frac{U_{1}^{2}}{2}\right)_{\xi}-f_{2}\left(V_{0}, U_{0}, \Theta_{0} ; V_{1}, U_{1}, \Theta_{1}\right)_{\xi} \\
& -\dot{\delta_{1}}\left(E_{0}+\frac{U_{0}^{2}}{2}\right)_{\xi}-\dot{\delta_{0}}\left(E_{1}+U_{0} U_{1}\right)_{\xi}-\left(E_{1}+U_{0} U_{1}\right)_{t}
\end{aligned}\right.
$$

and etc., where $\dot{s}=d s / d t, \dot{\delta_{0}}=d \delta_{0} / d t$, etc., and

$$
\begin{aligned}
& \mathcal{B}_{1}\left(V_{0}, U_{0} ; V_{1}, U_{1}\right)=-\frac{U_{1 \xi} V_{1}}{V_{0}^{2}}+\frac{U_{0 \xi} V_{1}^{2}}{V_{0}^{3}}, \\
& \mathcal{B}_{2}\left(V_{0}, U_{0} ; V_{1}, U_{1}\right)=-\frac{U_{0} U_{1 \xi} V_{1}}{V_{0}^{2}}+\frac{U_{0} U_{0 \xi} V_{1}^{2}}{V_{0}^{3}}+\frac{U_{1} U_{1 \xi}}{V_{0}}-\frac{U_{1} V_{1} U_{0 \xi}}{V_{0}^{2}},
\end{aligned}
$$

etc. The inner approximation is supposed to be valid in a small zone of size $O(\varepsilon)$ near the shock $x=s(t)$.

In a matching zone, we expect that the outer and the inner expansion agree with each other. Using the Taylor series to express the outer solutions in terms of $\xi$, we obtain the following "matching conditions" as $\xi \rightarrow \pm \infty$ :

$$
\begin{gathered}
H_{0}(\xi, t)=h_{0}(s(t) \pm 0, t)+o(1), \\
H_{1}(\xi, t)=h_{1}(s(t) \pm 0, t)+\left(\xi-\delta_{0}\right) \partial_{x} h_{0}(s(t) \pm 0, t)+o(1), \\
H_{2}(\xi, t)=h_{2}(s(t) \pm 0, t)+\left(\xi-\delta_{0}\right) \partial_{x} h_{1}(s(t) \pm 0, t)-\delta_{1} \partial_{x} h_{0}(s(t) \pm 0, t) \\
+\frac{1}{2}\left(\xi-\delta_{0}\right)^{2} \partial_{x}^{2} h_{0}(s(t) \pm 0, t)+o(1),
\end{gathered}
$$

and etc.

2.2. The structure of viscous shock profiles. Our construction of the approximate solutions depends on the properties of the forward traveling waves $H=(V, U, \Theta)^{t}$, which are the solutions of the following ordinary differential equations

$$
\left\{\begin{array}{l}
-\sigma V^{\prime}-U^{\prime}=0 \\
-\sigma U^{\prime}+p(V, \Theta)^{\prime}=\left(\frac{U^{\prime}}{V}\right)^{\prime}, \\
-\sigma\left(E+\frac{U^{2}}{2}\right)^{\prime}+(p(V, \Theta) U)^{\prime}=\left(\frac{\Theta^{\prime}}{V}+\frac{U U^{\prime}}{V}\right)^{\prime},
\end{array}\right.
$$

with the boundary conditions

$$
H(\xi) \rightarrow\left\{\begin{array}{l}
h_{l}=\left(v_{l}, u_{l}, \theta_{l}\right)^{t}, \quad \text { as } \xi \rightarrow-\infty, \\
h_{r}=\left(v_{r}, u_{r}, \theta_{r}\right)^{t}, \quad \text { as } \xi \rightarrow+\infty,
\end{array}\right.
$$


and moving with speed $\sigma$ satisfying

$$
\left\{\begin{array}{l}
-\sigma\left(v_{l}-v_{r}\right)-\left(u_{l}-u_{r}\right)=0 \\
-\sigma\left(u_{l}-u_{r}\right)+\left(p_{l}-p_{r}\right)=0 \\
-\sigma\left(e_{l}+\frac{u_{l}^{2}}{2}-\left(e_{r}+\frac{u_{r}^{2}}{2}\right)\right)+\left(p_{l} u_{l}-p_{r} u_{r}\right)=0
\end{array}\right.
$$

and the Lax's shock condition $\lambda_{3 r}<\sigma<\lambda_{3 l}$, where $p_{l}=p\left(v_{l}, \theta_{l}\right), e_{l}=e\left(\theta_{l}\right)$, etc.. Integrate the differential equations to get

$$
\left\{\begin{array}{l}
-\sigma V-U=a_{1} \\
\frac{U^{\prime}}{V}=-\sigma U+P+a_{2} \\
\frac{\Theta^{\prime}}{V}+\frac{U U^{\prime}}{V}=-\sigma\left(E+\frac{U^{2}}{2}\right)+P U+a_{3}
\end{array}\right.
$$

where $P=p(V, \Theta), E=e(\Theta), a_{1}=-\sigma v_{l}-u_{l}, a_{2}=\sigma u_{l}-p_{l}$ and $a_{3}=\sigma\left(e_{l}+\frac{u_{l}^{2}}{2}\right)-p_{l} u_{l}$. This system is transformed into

$$
\left\{\begin{array}{l}
U=-\sigma V-a_{1} \\
\frac{\sigma V^{\prime}}{V}=-\left\{P+\sigma^{2}\left(V-\frac{b_{1}}{\sigma^{2}}\right)\right\} \\
\frac{\Theta^{\prime}}{\sigma V}=-\left\{E-\frac{\sigma^{2}}{2}\left(V-\frac{b_{1}}{\sigma^{2}}\right)^{2}+\frac{b_{1}^{2}}{2 \sigma^{2}}-b_{2}\right\}
\end{array}\right.
$$

where $b_{1}=-\sigma a_{1}-a_{2}$ and $b_{2}=\frac{\sigma a_{1}^{2}+2 a_{1} a_{2}+2 a_{3}}{2 \sigma}$.

From [17], we know that there exists a shock profile $H=(V, U, \Theta)^{t}$, which connects the states $h_{l}$ and $h_{r}$. By a direct calculation[16], we can deduce that $H$ satisfies $\sigma V^{\prime}=-U^{\prime}>0, \sigma \Theta^{\prime}<0$ and

$$
\begin{aligned}
& \left|\partial_{\xi}(V, U)\right| \leq \bar{c}\left|v_{r}-v_{l}\right|,\left|\partial_{\xi}^{2}(V, U, \Theta)\right| \leq \bar{c}\left|v_{r}-v_{l}\right| \\
& \left|\partial_{\xi} \Theta\right| \leq \bar{c}(\gamma-1)\left|v_{r}-v_{l}\right| \text { and }\left|\partial_{\xi} \Theta\right| \leq \bar{c}(\gamma-1)\left|\partial_{\xi} V\right|
\end{aligned}
$$

where the constant $\bar{c}$ depends only on $h_{l}$. Moreover, as $\xi \rightarrow-\infty$,

$$
\begin{gathered}
\frac{\partial H}{\partial h_{l}}-I=O(1) e^{-\alpha|\xi|}, \\
\frac{\partial H}{\partial \sigma}=O(1) e^{-\alpha|\xi|} .
\end{gathered}
$$

As $\xi \rightarrow+\infty$,

$$
\begin{aligned}
& \frac{\partial H}{\partial h_{l}}-\frac{\partial h_{r}}{\partial h_{l}}=O(1) e^{-\alpha|\xi|}, \\
& \frac{\partial H}{\partial \sigma}-\frac{\partial h_{r}}{\partial \sigma}=O(1) e^{-\alpha|\xi|} .
\end{aligned}
$$


2.3. Solutions of the outer and inner problems. Now we construct $h_{j}$ and $H_{j}$ order by order.

The leading order outer function, $h_{0}$, is the single-shock solution in Theorem 1.2. For any fixed $\mathrm{t}$, the leading order inner solution $H_{0}(\xi, t)$ is exactly the viscous shock profile with $h_{l}(t) \equiv\left(v_{l}(t), u_{l}(t), \theta_{l}(t)\right)^{t}=h(s(t)-0, t), h_{r}(t) \equiv\left(v_{r}(t), u_{r}(t), \theta_{r}(t)\right)^{t}=$ $h(s(t)+0, t)$ and $\sigma=\dot{s}(t)$. So

$$
H_{0}(\xi, t)=H\left(\xi, h_{l}(t), \dot{s}(t)\right)
$$

Here we take the shift to be zero since it can be absorbed into $\delta_{0}(t)$.

Next we determine $h_{1}, H_{1}$ and $\delta_{0}(t)$ together. By the matching condition (2.12), we expect that

$$
H_{1}(\xi, t)=\xi \cdot \partial_{x} h_{0}(s(t) \pm 0, t)+O(1) \quad \text { as } \quad \xi \rightarrow \pm \infty
$$

So we set

$$
H_{1}(\xi, t)=\chi(\xi, t)+D(\xi, t)
$$

where $\chi(\xi, t)=\left(\chi_{1}, \chi_{2}, \chi_{3}\right)^{t}$ and $D(\xi, t)=\left(D_{1}, D_{2}, D_{3}\right)^{t}$ is a smooth function satisfying

$$
D(\xi, t)= \begin{cases}\xi \cdot \partial_{x} h_{0}(s(t)-0, t), & \xi<-1 \\ \xi \cdot \partial_{x} h_{0}(s(t)+0, t), & \xi>1\end{cases}
$$

Then inserting (2.22) into (2.9) and using (2.16)-(2.20), we obtain

$$
\left\{\begin{array}{l}
\dot{s} \chi_{1 \xi}+\chi_{2 \xi}=\dot{\delta_{0}} V_{\xi}+g_{1}(\xi, t) \\
\dot{s} \chi_{2 \xi}-\left(p_{v}(V, \Theta) \chi_{1}+p_{\theta}(V, \Theta) \chi_{3}\right)_{\xi}+\left(\frac{\chi_{2 \xi}}{V}-\frac{U_{\xi} \chi_{1}}{V^{2}}\right)_{\xi}=\dot{\delta_{0}} U_{\xi}+g_{2}(\xi, t) \\
\dot{s}\left(\frac{R}{\gamma-1} \chi_{3}+U \chi_{2}\right)_{\xi}-\left\{p(V, \Theta) \chi_{2}+\left(p_{v}(V, \Theta) \chi_{1}+p_{\theta}(V, \Theta) \chi_{3}\right) U\right\}_{\xi} \\
\quad+\left(\frac{\chi_{3 \xi}}{V}-\frac{\Theta}{V^{2}}\right)_{\xi}+\left(\frac{U_{\xi} \chi_{2}}{V}+\frac{U \chi_{2 \xi}}{V}-\frac{U U_{\xi} \chi_{1}}{V^{2}}\right)_{\xi}=\dot{\delta_{0}}\left(E+\frac{U^{2}}{2}\right)_{\xi}+g_{3}(\xi, t)
\end{array}\right.
$$

where $\left|g_{i}(\xi, t)\right| \leq c \exp \{-\alpha|\xi|\}$ for large $|\xi|, i=1,2,3$. Define $G_{i}(\xi, t)=\int_{0}^{\xi} g_{i}(\eta, t) d \eta$. Then we have

$$
\left\{\begin{array}{l}
\dot{s} \chi_{1}+\chi_{2}=\dot{\delta_{0}} V+G_{1}(\xi, t)+c_{1}(t), \\
\dot{s} \chi_{2}-\left(p_{v}(V, \Theta) \chi_{1}+p_{\theta}(V, \Theta) \chi_{3}\right)+\frac{\chi_{2 \xi}}{V}-\frac{U_{\xi} \chi_{1}}{V^{2}}=\dot{\delta_{0}} U+G_{2}(\xi, t)+c_{2}(t), \\
\dot{s}\left(\frac{R}{\gamma-1} \chi_{3}+U \chi_{2}\right)-\left\{p(V, \Theta) \chi_{2}+\left(p_{v}(V, \Theta) \chi_{1}+p_{\theta}(V, \Theta) \chi_{3}\right) U\right\} \\
\quad+\left(\frac{\chi_{3 \xi}}{V}-\frac{\Theta}{V^{2}} \chi_{1}\right. \\
\quad\left(\frac{U_{\xi} \chi_{2}}{V}+\frac{U \chi_{2 \xi}}{V}-\frac{U U_{\xi} \chi_{1}}{V^{2}}\right)=\dot{\delta_{0}}\left(E+\frac{U^{2}}{2}\right)+G_{3}(\xi, t)+c_{3}(t),
\end{array}\right.
$$

where $c_{i}(t) \in R^{1}, i=1,2,3$, are integration constants to be determined later. Letting 
$\xi \rightarrow \pm \infty$ and using the matching condition (2.12), we obtain

$$
\left\{\begin{array}{c}
\left((\gamma-1) p_{l}-\dot{s}^{2} v_{l}\right)\left(\dot{\delta_{0}} v_{l}+c_{1}(t)+G_{1-}\right)+\left(\dot{s} v_{l}-(\gamma-1) u_{l}\right)\left(\dot{\delta}_{0} u_{l}+c_{2}(t)+G_{2-}\right) \\
\quad+(\gamma-1)\left(\dot{\dot{\delta}_{0}}\left(\frac{R}{\gamma-1} \theta_{l}+\frac{u_{l}^{2}}{2}\right)+c_{3}(t)+G_{3-}\right)=\dot{s}\left(\gamma p_{l}-\dot{s}^{2} v_{l}\right)\left(v_{1}^{l}-\delta_{0} \partial_{x} v_{l}\right), \\
p_{l}\left(\dot{\delta_{0}} v_{l}+c_{1}(t)+G_{1-}\right)+\left((\gamma-1) u_{l}-\dot{s} v_{l}\right)\left(\dot{\delta_{0}} u_{l}+c_{2}(t)+G_{2-}\right) \\
\quad-(\gamma-1)\left(\dot{\delta_{0}}\left(\frac{R}{\gamma-1} \theta_{l}+\frac{u_{l}^{2}}{2}\right)+c_{3}(t)+G_{3-}\right)=\left(\gamma p_{l}-\dot{s}^{2} v_{l}\right)\left(u_{1}^{l}-\delta_{0} \partial_{x} u_{l}\right), \\
(\gamma-1)\left\{p_{l}^{2}\left(\dot{\delta_{0}} v_{l}+c_{1}(t)+G_{1-}\right)-\left(\dot{s} v_{l} p_{l}+\left(p_{l}-\dot{s}^{2} v_{l}\right) u_{l}\right)\left(\dot{\delta_{0}} u_{l}+c_{2}(t)+G_{2-}\right)\right. \\
\left.\quad+\left(p_{l}-\dot{s}^{2} v_{l}\right)\left(\dot{\delta_{0}}\left(\frac{R}{\gamma-1} \theta_{l}+\frac{u_{l}^{2}}{2}\right)+c_{3}(t)+G_{3-}\right)\right\}=R \dot{s}\left(\gamma p_{l}-\dot{s}^{2} v_{l}\right)\left(\theta_{1}^{l}-\delta_{0} \partial_{x} \theta_{l}\right),
\end{array}\right.
$$

and

$$
\left\{\begin{array}{c}
\left((\gamma-1) p_{r}-\dot{s}^{2} v_{r}\right)\left(\dot{\delta_{0}} v_{r}+c_{1}(t)+G_{1+}\right)+\left(\dot{s} v_{r}-(\gamma-1) u_{r}\right)\left(\dot{\delta_{0}} u_{r}+c_{2}(t)+G_{2+}\right) \\
\quad+(\gamma-1)\left(\dot{\delta_{0}}\left(\frac{R}{\gamma-1} \theta_{r}+\frac{u_{r}^{2}}{2}\right)+c_{3}(t)+G_{3+}\right)=\dot{s}\left(\gamma p_{r}-\dot{s}^{2} v_{r}\right)\left(v_{1}^{r}-\delta_{0} \partial_{x} v_{r}\right), \\
p_{r}\left(\dot{\delta_{0}} v_{r}+c_{1}(t)+G_{1+}\right)+\left((\gamma-1) u_{r}-\dot{s} v_{r}\right)\left(\dot{\delta_{0}} u_{r}+c_{2}(t)+G_{2+}\right) \\
\quad-(\gamma-1)\left(\dot{\delta_{0}}\left(\frac{R}{\gamma-1} \theta_{r}+\frac{u_{r}^{2}}{2}\right)+c_{3}(t)+G_{3+}\right)=\left(\gamma p_{r}-\dot{s}^{2} v_{r}\right)\left(u_{1}^{r}-\delta_{0} \partial_{x} u_{r}\right), \\
(\gamma-1)\left\{p_{r}^{2}\left(\dot{\delta_{0}} v_{r}+c_{1}(t)+G_{1+}\right)-\left(\dot{s} v_{r} p_{r}+\left(p_{r}-\dot{s}^{2} v_{r}\right) u_{r}\right)\left(\dot{\delta_{0}} u_{r}+c_{2}(t)+G_{2+}\right)\right. \\
\left.\quad+\left(p_{r}-\dot{s}^{2} v_{r}\right)\left(\dot{R} \dot{\delta}_{0}\left(\frac{R}{\gamma-1} \theta_{r}+\frac{u_{r}^{2}}{2}\right)+c_{3}(t)+G_{3+}\right)\right\}=R \dot{s}\left(\gamma p_{r}-\dot{s}^{2} v_{r}\right)\left(\theta_{1}^{r}-\delta_{0} \partial_{x} \theta_{r}\right) .
\end{array}\right.
$$

Write $\beta_{\text {in }} \equiv\left\{v_{1}^{r}, u_{1}^{r}, \theta_{1}^{r}, \theta_{1}^{l}\right\}$ and $\beta_{\text {out }} \equiv\left\{v_{1}^{l}, u_{1}^{l}\right\}$. We first consider (2.25). Since the determinant of the Jacobian matrix

$$
\operatorname{det} J=-\frac{(\gamma-1) \dot{s}}{v_{r}}\left(\gamma v_{r} p_{r}-\dot{s}^{2} v_{r}^{2}\right) \neq 0,
$$

we can solve $\dot{\delta_{0}} v_{r}+c_{1}(t), \dot{\delta_{0}} u_{r}+c_{2}(t)$ and $\dot{\delta_{0}}\left(\frac{R}{\gamma-1} \theta_{r}+\frac{u_{r}^{2}}{2}\right)+c_{3}(t)$ from $(2.25)$ in terms of the terms on the right-hand side of (2.25). Then substituting the resulting expression into the last equation of (2.24), we arrive at the ordinary differential equation for $\delta_{0}$ :

$$
\dot{\delta}_{0}+E_{1}(t) \delta_{0}=E_{21}(t) \partial_{x} v_{1}^{r}+E_{22}(t) \partial_{x} u_{1}^{r}+E_{23}(t) \partial_{x} \theta_{1}^{r}+E_{24}(t) \partial_{x} \theta_{1}^{l}+F(t),
$$

provided that $(\gamma-1) \mu$ is suitably small. Here $E_{1}(t), E_{2 j}(t)$ and $F(t)$ are some known smooth functions, and $E_{1}(t)$ and $E_{2 j}(t)$ remain bounded even as $\mu \rightarrow 0,1 \leq j \leq 4$. Solving for $\delta_{0}$ from $(2.26)$ up to a constant, we obtain $c(t)$ uniquely in terms of $\beta_{i n}$. Then substituting the expression of $\delta_{0}$ and $c(t)$ into the first two equations of (2.24), we can express $v_{1}^{l}, u_{1}^{l}$ in terms of $\beta_{i n}$. Then the theory of linear hyperbolic equations $[3,4]$ shows that the problem $(2.3),(2.26)$ has a solution smooth up to the shock provided that the initial value, $h_{1}(x, 0)$, is chosen to satisfy the appropriate compatibility conditions at $x=s(0)$. Thus $h_{1}(x, t)$ is completely determined, which in turn gives $\delta_{0}$ and $c(t)$ by (2.24)-(2.26), and therefore $H_{1}(\xi, t)$. Now we summarize the above discussion to achieve

Proposition 2.1. If $(\gamma-1) \mu$ is suitably small, then $h_{1}(x, t), H_{1}(\xi, t)$ and $\delta_{0}$ can be established such that

(i) $h_{1}(x, t)$ and its derivatives are uniformly continuous up to $x=s(t)$, and

$$
\sum_{0 \leq k \leq 2 n+1} \int_{0}^{T} \int\left|\partial_{x}^{k} h_{1}(x, t)\right|^{2} d x d t<\infty .
$$


(ii) $H_{1}(\xi, t)$ and $\delta_{0}$ are smooth functions, and there is an $\alpha>0$ such that as $\xi \rightarrow \pm \infty$

$$
H_{1}(\xi, t)=h_{1}(s(t) \pm 0, t)+\left(\xi-\delta_{0}\right) \partial_{x} h_{0}(s(t) \pm 0, t)+O(1) \exp \{-\alpha|\xi|\} .
$$

The above constructions can be carried out to any order. In particular, we can determine $h_{2}, H_{2}, \delta_{1} ; \cdots ; h_{n}, H_{n}$ and $\delta_{n-1}$ for $n \geq 3$ and the similar results as in Proposition 2.1 hold for them.

2.4. Approximate solutions. Now we can construct an approximate solution to (1.1) by patching the truncated outer and inner solutions in the previous discussion. For $n \geq 3$, define

$$
I(x, t)=H\left(\frac{x-s(t)}{\varepsilon}+\sum_{j=0}^{n-1} \varepsilon^{j} \delta_{j}(t), t\right)+\sum_{i=1}^{n} \varepsilon^{i} H_{i}\left(\frac{x-s(t)}{\varepsilon}+\sum_{j=0}^{n-1} \varepsilon^{j} \delta_{j}(t), t\right),
$$

and

$$
O(x, t)=\sum_{i=0}^{n} \varepsilon^{i} h_{i}(x, t)
$$

Let $m \in C_{0}^{\infty}(R)$ satisfy $0 \leq m(y) \leq 1$, and

$$
m(y)= \begin{cases}1, & |y| \leq 1 \\ 0, & |y| \geq 2\end{cases}
$$

Set $\nu \in\left(\frac{1}{2}, 1\right)$ to be a constant. Then we define the approximate solution to (1.1) as

$$
S^{\varepsilon}(x, t)=m\left(\frac{x-s(t)}{\varepsilon^{\nu}}\right) I(x, t)+\left(1-m\left(\frac{x-s(t)}{\varepsilon^{\nu}}\right)\right) O(x, t)+d(x, t),
$$

where $d(x, t)$ is a higher-order correction term to be determined. We use the following notations:

$$
S^{\varepsilon}=\left(\overline{v^{\varepsilon}}, \overline{u^{\varepsilon}}, \overline{\theta^{\varepsilon}}\right)^{t}, \quad I=\left(I_{1}, I_{2}, I_{3}\right)^{t}, \quad O=\left(O_{1}, O_{2}, O_{3}\right)^{t}, \quad d=\left(d_{1}, d_{2}, d_{3}\right)^{t} .
$$

Using the structures of the various orders of inner and outer solutions, we compute that

$$
\left\{\begin{array}{l}
\overline{v_{t}^{\varepsilon}}-\bar{u}_{x}^{\varepsilon}=d_{1 t}-d_{2 x}+q_{1}(x, t), \\
\overline{u_{t}^{\varepsilon}}+p\left(\overline{v^{\varepsilon}}, \overline{\theta^{\varepsilon}}\right)-\varepsilon\left(\frac{\bar{u}_{x}^{\varepsilon}}{v^{\varepsilon}}\right)_{x}=d_{2 t}-\varepsilon\left(\frac{d_{2 x}}{B_{1}}\right)_{x}+\sum_{i=2}^{4} q_{i}(x, t)-q_{5 x}(x, t)+q_{6 x}(x, t), \\
\left(e\left(\overline{\theta^{\varepsilon}}\right)+\frac{\bar{u}^{\varepsilon^{2}}}{2}\right)_{t}+\left(p\left(\overline{v^{\varepsilon}}, \overline{\theta^{\varepsilon}}\right) \overline{u^{\varepsilon}}\right)_{x}-\kappa\left(\frac{\bar{\theta}_{x}^{\varepsilon}}{\bar{v}^{\varepsilon}}\right)_{x}-\varepsilon\left(\frac{\bar{u}^{\varepsilon}}{\overline{v^{\varepsilon}}}\right)_{x} \\
=\frac{R}{\gamma-1} d_{3 t}-\varepsilon\left(\frac{d_{3 x}}{B_{1}}\right)_{x}+\sum_{j=7}^{10} q_{j}(x, t)-q_{11 x}(x, t)+q_{12 x}(x, t) .
\end{array}\right.
$$

Here

$$
\begin{gathered}
q_{1}(x, t)=m_{t}\left(I_{1}-O_{1}\right)-m_{x}\left(I_{2}-O_{2}\right)+m\left(I_{1 t}-I_{2 x}\right) \\
q_{2}(x, t)=m_{t}\left(I_{2}-O_{2}\right)+\left\{p\left(B_{1}, B_{3}\right)-m p\left(I_{1}, I_{3}\right)-(1-m) p\left(O_{1}, O_{3}\right)\right\}_{x}
\end{gathered}
$$




$$
\begin{aligned}
& +m_{x}\left(p\left(I_{1}, I_{3}\right)-p\left(O_{1}, O_{3}\right)\right)-\varepsilon m_{x}\left(\frac{I_{2 x}}{I_{1}}-\frac{O_{2 x}}{O_{1}}\right) \\
& q_{3}(x, t)=m\left\{\left(p\left(I_{1}, I_{3}\right)-\Gamma\left(p\left(I_{1}, I_{3}\right)\right)\right)_{x}-\varepsilon\left(\frac{I_{2 x}}{I_{1}}-\Gamma\left(\frac{I_{2 x}}{I_{1}}\right)\right)_{x}\right. \\
& \left.+\varepsilon^{n} U_{n t}+\varepsilon^{n+1}\left(\sum_{i=0}^{n-1} \sum_{j=i}^{n-1} \varepsilon^{i} \delta_{j} U_{n+i-j}\right)_{x}\right\}, \\
& q_{4}(x, t)=(1-m)\left\{\left(p\left(O_{1}, O_{3}\right)-\Gamma\left(p\left(O_{1}, O_{3}\right)\right)\right)_{x}-\varepsilon\left(\frac{O_{2 x}}{O_{1}}-\Gamma\left(\frac{O_{2 x}}{O_{1}}\right)\right)_{x}\right\} \\
& q_{5}(x, t)=\varepsilon\left\{\frac{1}{\overline{v^{\varepsilon}}} B_{2 x}-m \frac{I_{2 x}}{I_{1}}-(1-m) \frac{O_{2 x}}{O_{1}}+\left(\frac{1}{\overline{v^{\varepsilon}}}-\frac{1}{B_{1}}\right) d_{2 x}\right\}, \\
& q_{6}(x, t)=p\left(\overline{v^{\varepsilon}}, \overline{\theta^{\varepsilon}}\right)-p\left(B_{1}, B_{3}\right) \\
& q_{7}(x, t)=\frac{R}{\gamma-1} m_{t}\left(I_{3}-O_{3}\right)+m m_{t}\left(I_{2}-O_{2}\right)^{2}-m(1-m)\left(I_{2}-O_{2}\right)\left(I_{2}-O_{2}\right)_{t} \\
& +m_{t} O_{2}\left(I_{2}-O_{2}\right)+m_{x}\left(p\left(I_{1}, I_{3}\right) I_{2}-p\left(O_{1}, O_{3}\right) O_{2}\right) \\
& +\left\{p\left(B_{1}, B_{3}\right) B_{2}-m\left(p\left(I_{1}, I_{3}\right) I_{2}-(1-m)\left(p\left(O_{1}, O_{3}\right) O_{2}\right\}_{x}\right.\right. \\
& -\varepsilon m_{x}\left(\frac{I_{3 x}}{I_{1}}-\frac{O_{3 x}}{O_{1}}+\frac{I_{2} I_{2 x}}{I_{1}}-\frac{O_{2} O_{2 x}}{O_{1}}\right), \\
& q_{8}(x, t)=m\left\{\left[p\left(I_{1}, I_{3}\right) I_{2}-\Gamma\left(p\left(I_{1}, I_{3}\right) I_{2}\right)\right]_{x}-\varepsilon\left[\frac{I_{3 x}}{I_{1}}-\Gamma\left(\frac{I_{3 x}}{I_{1}}\right)+\frac{I_{2} I_{2 x}}{I_{1}}-\Gamma\left(\frac{I_{2} I_{2 x}}{I_{1}}\right)\right]_{x}\right. \\
& +\varepsilon^{n}\left(E_{n t}+\frac{1}{2} \sum_{i=0}^{n} \sum_{j=i}^{n} \varepsilon^{i} U_{j} U_{n+i-j}\right)_{t} \\
& +\varepsilon^{n+1}\left(\sum_{i=0}^{n-1} \sum_{j=i}^{n-1} \varepsilon^{i} \delta_{j} E_{n+i-j}+\frac{1}{2} \sum_{i=0}^{n-1} \sum_{j=i}^{n+i} \sum_{k=j-i}^{n} \delta_{i} \varepsilon^{j} U_{k} U_{n-i+j-k}\right. \\
& \left.\left.+\frac{1}{2} \sum_{i=1}^{n-1} \sum_{j=0}^{i-1} \sum_{k=0}^{n-i+j} \delta_{i} \varepsilon^{j} U_{k} U_{n-i+j-k}-\frac{\dot{s}}{2} \sum_{i=0}^{n-1} \sum_{j=i+1}^{n} \varepsilon^{i} U_{j} E_{n+1+i-j}\right)_{x}\right\}, \\
& q_{9}(x, t)=(1-m)\left\{\left[p\left(O_{1}, O_{3}\right) O_{2}-\Gamma\left(p\left(O_{1}, O_{3}\right) O_{2}\right)\right]_{x}-\varepsilon\left[\frac{O_{3 x}}{O_{1}}-\Gamma\left(\frac{O_{3 x}}{O_{1}}\right)+\frac{O_{2} O_{2 x}}{O_{1}}\right.\right. \\
& \left.\left.-\Gamma\left(\frac{O_{2} O_{2 x}}{O_{1}}\right)\right]_{x}+\frac{1}{2} \varepsilon^{n+1}\left(\sum_{i=0}^{n-1} \sum_{j=i+1}^{n} \varepsilon^{i} u_{j} u_{n+1+i-j}\right)_{t}\right\}, \\
& q_{10}(x, t)=d_{2} \overline{u_{t}^{\varepsilon}}+d_{2 t} \overline{u^{\varepsilon}}-\varepsilon\left(\frac{d_{2 x}}{B_{1}} \bar{u}^{\varepsilon}\right)_{x},
\end{aligned}
$$




$$
\begin{aligned}
q_{11}(x, t)= & \varepsilon\left\{\left(\frac{1}{\bar{v}^{\varepsilon}} B_{3 x}-m \frac{I_{3 x}}{I_{1}}-(1-m) \frac{O_{3 x}}{O_{1}}\right)+\left(\frac{1}{\bar{v}^{\varepsilon}}-\frac{1}{B_{1}}\right) d_{3 x}+\left(\frac{\bar{u}^{\varepsilon}}{\bar{v}^{\varepsilon}} B_{2 x}-m \frac{I_{2} I_{2 x}}{I_{1}}\right.\right. \\
- & \left.\left.(1-m) \frac{O_{2} O_{2 x}}{O_{1}}\right)+\left(\frac{\overline{u^{\varepsilon}}}{\bar{v}^{\varepsilon}}-\frac{\bar{u}^{\bar{\varepsilon}}}{B_{1}}\right) d_{2 x}\right\} \\
q_{12}(x, t) & =p\left(\bar{v}^{\bar{\varepsilon}}, \bar{\theta}^{\bar{\varepsilon}}\right) \bar{u}^{\varepsilon}-p\left(B_{1}, B_{3}\right) B_{2}
\end{aligned}
$$

where $B_{j}=m I_{j}+(1-m) O_{j}, j=1,2,3 ; \Gamma\left(p\left(I_{1}, I_{3}\right)\right), \Gamma\left(\frac{I_{2 x}}{I_{1}}\right), \Gamma\left(p\left(I_{1}, I_{3}\right) I_{2}\right), \Gamma\left(\frac{I_{3 x}}{I_{1}}\right)$ and $\Gamma\left(\frac{I_{2} I_{2 x}}{I_{1}}\right)$ denote the truncated Taylor's expansion of $p\left(I_{1}, I_{3}\right), \frac{I_{2 x}}{I_{1}}, p\left(I_{1}, I_{3}\right) I_{2}, \frac{I_{3 x}}{I_{1}}$ and $\frac{I_{2} I_{2 x}}{I_{1}}$, respectively, at $(V, U, \Theta)$, including all the terms of the orders $O(1) \varepsilon^{k}, 0 \leq$ $k \leq n ; \Gamma\left(p\left(O_{1}, O_{3}\right)\right), \Gamma\left(\frac{O_{2 x}}{O_{1}}\right), \Gamma\left(p\left(O_{1}, O_{3}\right) O_{2}\right), \Gamma\left(\frac{O_{3 x}}{O_{1}}\right)$ and $\Gamma\left(\frac{O_{2} O_{2 x}}{O_{1}}\right)$ denote the truncated Taylor's expansion of $p\left(O_{1}, O_{3}\right), \frac{O_{2 x}}{O_{1}}, p\left(O_{1}, O_{3}\right) O_{2}, \frac{O_{3 x}}{O_{1}}$ and $\frac{O_{2} O_{2 x}}{O_{1}}$, respectively, at $\left(v_{0}, u_{0}, \theta_{0}\right)$, including all the terms of the orders $O(1) \varepsilon^{k}, 0 \leq k \leq n$.

In view of our construction, we have

i) $\operatorname{supp}\left(q_{1}, q_{3}, q_{8}\right) \subseteq\left\{(x, t):|x-s(t)| \leq 2 \varepsilon^{\nu}, 0 \leq t \leq T\right\}$, and

$$
\partial_{x}^{l}\left(q_{1}, q_{3}, q_{8}\right)(x, t)=O(1) \varepsilon^{(n-l) \nu}, \quad l=0,1,2,3 .
$$

ii) $\operatorname{supp}\left(q_{2}, q_{7}\right) \subseteq\left\{(x, t): \varepsilon^{\nu} \leq|x-s(t)| \leq 2 \varepsilon^{\nu}, 0 \leq t \leq T\right\}$, and

$$
\partial_{x}^{l}\left(q_{2}, q_{7}\right)(x, t)=O(1) \varepsilon^{(n-l) \nu}, \quad l=0,1,2,3 .
$$

iii) $\operatorname{supp}\left(q_{4}, q_{9}\right) \subseteq\left\{(x, t):|x-s(t)| \geq \varepsilon^{\nu}, 0 \leq t \leq T\right\}$, and

$$
\begin{aligned}
& \partial_{x}^{l}\left(q_{4}, q_{9}\right)(x, t)=O(1) \varepsilon^{n+1-l \nu}, \quad\left(\int_{0}^{T}\left\|\left(q_{4}, q_{9}\right)(\cdot, t)\right\|^{2} d t\right)^{\frac{1}{2}} \leq O(1) \varepsilon^{n+1}, \\
& \left(\int_{0}^{T}\left\|\partial_{x}^{l}\left(q_{4}, q_{9}\right)(\cdot, t)\right\|^{2} d t\right)^{\frac{1}{2}} \leq O(1) \varepsilon^{n+1-\left(l-\frac{1}{2}\right) \nu}, \quad l=1,2,3 .
\end{aligned}
$$

We now choose $d(x, t)=\left(d_{1}(x, t), d_{2}(x, t), d_{3}(x, t)\right)$ to be the solution of

$$
\left\{\begin{array}{l}
d_{1 t}-d_{2 x}=-q_{1}(x, t) \\
d_{2 t}-\varepsilon\left(\frac{d_{2 x}}{B_{1}}\right)_{x}=-\sum_{i=2}^{4} q_{i}(x, t) \\
\frac{R}{\gamma-1} d_{3 t}-\varepsilon\left(\frac{d_{3 x}}{B_{1}}\right)_{x}=-\sum_{j=7}^{10} q_{j}(x, t) \\
d_{1}(x, 0)=d_{2}(x, 0)=d_{3}(x, 0)=0
\end{array}\right.
$$

so that $S^{\varepsilon}$ satisfies

$$
\left\{\begin{array}{l}
\overline{v_{t}^{\varepsilon}}-\overline{u_{x}^{\varepsilon}}=0, \\
\overline{u_{t}^{\varepsilon}}+p\left(\overline{v^{\varepsilon}}, \overline{\theta^{\varepsilon}}\right)_{x}=\varepsilon\left(\frac{\overline{u_{x}^{\varepsilon}}}{\bar{v}^{\bar{\varepsilon}}}\right)_{x}-q_{5 x}(x, t)+q_{6 x}(x, t), \\
\left(e\left(\overline{\theta^{\varepsilon}}\right)+\frac{\bar{u}^{\varepsilon}}{2}\right)_{t}+\left(p\left(\bar{v}^{\bar{\varepsilon}}, \bar{\theta}^{\varepsilon}\right) \bar{u}^{\varepsilon}\right)_{x}=\kappa\left(\frac{\overline{\theta_{x}^{\varepsilon}}}{\bar{v}^{\bar{\varepsilon}}}\right)_{x}+\varepsilon\left(\frac{\overline{u^{\varepsilon}} \bar{u}_{x}^{\varepsilon}}{v^{\varepsilon}}\right)_{x}-q_{11 x}(x, t)+q_{12 x}(x, t) .
\end{array}\right.
$$

Since $B_{1}(x, t)>0$ is bounded below and above, and uniformly continuous, by the result of [14], $(2.48)_{2,3}$ admit fundamental solutions $G_{2}(x, t)$ and $G_{3}(x, t)$, respectively, which are bounded as follows:

$$
\left|G_{2}(x, t), G_{3}(x, t)\right| \leq k_{1}(\varepsilon t)^{-\frac{1}{2}} e^{-\frac{k_{2}}{\varepsilon t}}, \quad \forall t \in[0, T],
$$


where the constants $k_{1}$ and $k_{2}$ depend only on the lower and upper bounds of $B_{1}$ and $T$. By the same method of [2] and a direct calculation, and the fact $d_{1}(x, t)=$ $\int_{0}^{t} d_{2 x}(x, \tau) d \tau-\int_{0}^{t} q_{1}(x, \tau) d \tau$, we have the following results. Here we omit the proof.

Lemma 2.2. Let $d(x, t)$ be the solution of (2.48). The following estimates hold for all $t \in[0, T]$ :

i)

$$
\begin{aligned}
& \left\|\partial_{x}^{l} d_{2}(\cdot, t)\right\|_{L^{\infty}} \leq O(1) \varepsilon^{(n+1-l) \nu-\frac{1}{2}}, \quad \text { for } \quad l=0,1,2,3,4, \\
& \left\|d_{2}(\cdot, t)\right\| \leq O(1) \varepsilon^{(n+1) \nu+\frac{\alpha}{2}-\frac{1}{2}}, \quad \alpha \in\left(0, \frac{1}{2}\right), \\
& \left\|\partial_{x}^{l} d_{2}(\cdot, t)\right\| \leq O(1) \varepsilon^{\left(n+1-l+\frac{1}{2}\right) \nu-\frac{1}{2}}, \quad l=1,2,3,4 .
\end{aligned}
$$

ii)

$$
\left\|\partial_{x}^{l} d_{1}(\cdot, t)\right\|_{L^{\infty}} \leq O(1) \varepsilon^{(n-l) \nu-\frac{1}{2}}, \quad \text { for } l=0,1,2,3,
$$

$$
\left\|\partial_{x}^{l} d_{1}(\cdot, t)\right\| \leq O(1) \varepsilon^{\left(n-l+\frac{1}{2}\right) \nu-\frac{1}{2}}, \quad l=0,1,2,3 .
$$

$$
\left\|\partial_{x}^{l} q_{10}(\cdot, t)\right\|_{L^{\infty}} \leq O(1) \varepsilon^{(n-l) \nu-\frac{1}{2}}, \quad \text { for } \quad l=0,1,2,3,
$$

$\left\|\partial_{x}^{l} q_{10}(\cdot, t)\right\| \leq O(1) \varepsilon^{\left(n-l+\frac{1}{2}\right) \nu-\frac{1}{2}}, \quad l=0,1,2,3$.

$i v)$

$$
\left\|\partial_{x}^{l} d_{3}(\cdot, t)\right\|_{L^{\infty}} \leq O(1) \varepsilon^{(n-l) \nu-\frac{1}{2}}, \quad \text { for } \quad l=0,1,2,3
$$

$$
\left\|\partial_{x}^{l} d_{3}(\cdot, t)\right\| \leq O(1) \varepsilon^{\left(n-l+\frac{1}{2}\right) \nu-\frac{1}{2}}, \quad l=0,1,2,3 .
$$

v) $\quad\left\|\partial_{x}^{l}\left(q_{5}, q_{6}, q_{11}, q_{12}\right)(\cdot, t)\right\| \leq O(1) \varepsilon^{\left(n+\frac{1}{2}\right) \nu-l-\frac{1}{2}}, \quad l=0,1,2,3$.

It follows from our construction that $S^{\varepsilon}$ has the following property.

Lemma 2.3. Let $S^{\varepsilon}$ be defined in (2.31), then

$$
S^{\varepsilon}(x, t)= \begin{cases}h_{0}(x, t)+O(1) \varepsilon, & \text { for }|x-s(t)| \geq \varepsilon^{\nu} \\ H_{0}(\xi, t)+O(1) \varepsilon^{\nu}, & \text { for }|x-s(t)| \leq 2 \varepsilon^{\nu} .\end{cases}
$$

Under the following coordinate transformation

$$
y=\frac{x-s(t)}{\varepsilon}, \quad \tau=\frac{t}{\varepsilon},
$$

we have

$$
\partial_{y}^{l} S^{\varepsilon}=m \partial_{y}^{l} H_{0}+O(1) \varepsilon, \quad \partial_{\tau} S^{\varepsilon}=O(1) \varepsilon, \quad 1 \leq l \leq 3 .
$$

3. Stability analysis. We now show that there exists an exact solution to (1.1) in a neighborhood of the approximate solution $S^{\varepsilon}(x, t)$, and that the asymptotic behavior of the viscous solution is given by $S^{\varepsilon}$ for small viscosity $\varepsilon$.

Suppose that $h^{\varepsilon}=\left(v^{\varepsilon}, u^{\varepsilon}, \theta^{\varepsilon}\right)$ is the exact solution to (1.1) with the initial data $h^{\varepsilon}(x, 0)=S^{\varepsilon}(x, 0)$. We decompose the solution as

$$
\begin{aligned}
v^{\varepsilon}(x, t) & =\bar{v}^{\varepsilon}(x, t)+\phi(x, t), \\
u^{\varepsilon}(x, t) & =\bar{u}^{\varepsilon}(x, t)+\psi(x, t), \\
\theta^{\varepsilon}(x, t) & =\bar{\theta}^{\varepsilon}(x, t)+\zeta(x, t), \\
\left(e\left(\theta^{\varepsilon}\right)+\frac{\left(u^{\varepsilon}\right)^{2}}{2}\right)(x, t) & =\left(e\left(\bar{\theta}^{\varepsilon}\right)+\frac{\bar{u}^{\varepsilon}}{2}\right)(x, t)+w(x, t),
\end{aligned}
$$


for $(x, t) \in R \times[0, T]$. Then using the relation (2.49) for $S^{\varepsilon}$, we obtain that

$$
\left\{\begin{array}{l}
\phi_{t}-\psi_{x}=0, \\
\psi_{t}+\left(\overline{p_{v}} \phi+\overline{p_{\theta}} \zeta\right)_{x}+Q_{1}\left(\overline{v^{\varepsilon}}, \overline{\theta^{\varepsilon}} ; \phi, \psi\right)_{x}=\varepsilon\left(\frac{u_{x}^{\varepsilon}}{v^{\varepsilon}}-\frac{\overline{u_{x}^{\varepsilon}}}{\bar{v}^{\varepsilon}}\right)_{x}+\left(q_{5}-q_{6}\right)_{x}, \\
w_{t}+\left(\overline{u^{\varepsilon}} \overline{p_{v} \phi}+\bar{p} \psi+\bar{u}^{\varepsilon} \overline{p_{\theta}} \zeta\right)_{x}+Q_{2}\left(\overline{v^{\varepsilon}}, \overline{u^{\varepsilon}}, \bar{\theta}^{\varepsilon} ; \phi, \psi, \zeta\right)_{x} \\
=\kappa\left(\frac{\theta_{x}^{\varepsilon}}{v^{\varepsilon}}-\frac{\bar{\theta}_{x}^{\varepsilon}}{\bar{v}^{\varepsilon}}\right)_{x}+\varepsilon\left(\frac{u^{\varepsilon} u_{x}^{\varepsilon}}{v^{\varepsilon}}-\frac{\bar{u}^{\varepsilon} \bar{u}_{x}^{\varepsilon}}{\bar{v}^{\varepsilon}}\right)_{x}+\left(q_{11}-q_{12}\right)_{x}, \\
\phi(x, 0)=\psi(x, 0)=w(x, 0)=0
\end{array}\right.
$$

where

$$
\begin{aligned}
& \bar{p}=p\left(\overline{v^{\varepsilon}}, \overline{\theta^{\varepsilon}}\right), \overline{p_{v}}=p_{v}\left(\bar{v}^{\varepsilon}, \overline{\theta^{\varepsilon}}\right), \overline{p_{\theta}}=p_{\theta}\left(\overline{v^{\varepsilon}}, \overline{\theta^{\varepsilon}}\right), \\
& Q_{1}=p\left(v^{\varepsilon}, \theta^{\varepsilon}\right)-p\left(\overline{v^{\varepsilon}}, \overline{\theta^{\varepsilon}}\right)-\left(\overline{p_{v}} \phi+\overline{p_{\theta}} \zeta\right) \text { satisfies }\left|Q_{1}\right| \leq O(1)\left(|\phi|^{2}+|\zeta|^{2}\right), \\
& Q_{2}=p\left(v^{\varepsilon}, \theta^{\varepsilon}\right) u^{\varepsilon}-\bar{p} \bar{u}^{\varepsilon}-\left(\overline{u^{\varepsilon}} \overline{p_{v}} \phi+\bar{p} \psi+\overline{u^{\varepsilon}} \overline{p_{\theta}} \zeta\right) \\
& \quad \text { satisfies }\left|Q_{2}\right| \leq O(1)\left(|\phi|^{2}+|\psi|^{2}+|\zeta|^{2}\right) .
\end{aligned}
$$

To exploit the fact that a shock satisfying the entropy condition is compressive, we need to integrate the system $(3.2)$ once. Thus we set $(\phi, \psi, w)(x, t)=$ $\left(\bar{\Phi}_{x}, \bar{\Psi}_{x}, \tilde{W}_{x}\right)(x, t)$ and $\bar{W}=\frac{\gamma-1}{R}\left(\tilde{W}-\bar{u}^{\varepsilon} \bar{\Psi}\right)$. Then

$$
\zeta=\bar{W}_{x}+\frac{\gamma-1}{R}\left(\bar{u}_{x}^{\varepsilon} \bar{\Psi}-\frac{1}{2} \bar{\Psi}_{x}^{2}\right) \text { and } w=\frac{R}{\gamma-1} \bar{W}_{x}+(\bar{u} \bar{\Psi})_{x} .
$$

Substitute these quantities into (3.2) and integrate the resulting equation with respect to $x$ to obtain

$$
\left\{\begin{array}{l}
\bar{\Phi}_{t}-\bar{\Psi}_{x}=0 \\
\bar{\Psi}_{t}+\overline{p_{v}} \phi+\overline{p_{\theta}} \zeta+Q_{1}=\varepsilon\left(\frac{u_{x}^{\varepsilon}}{v^{\varepsilon}}-\frac{\overline{u_{x}^{\varepsilon}}}{\bar{v}^{\varepsilon}}\right)+q_{5}-q_{6} \\
\left(\frac{R}{\gamma-1} \bar{W}+\overline{u^{\varepsilon}} \bar{\Psi}\right)_{t}+\left(\overline{u^{\varepsilon}} \overline{p_{v}} \phi+\bar{p} \psi+\bar{u}^{\varepsilon} \overline{\theta_{\theta}} \zeta\right)+Q_{2} \\
=\kappa\left(\frac{\theta_{x}^{\varepsilon}}{v^{\varepsilon}}-\frac{\overline{\theta_{x}^{\varepsilon}}}{\bar{v}^{\varepsilon}}\right)+\varepsilon\left(\frac{u^{\varepsilon} u_{x}^{\varepsilon}}{v^{\varepsilon}}-\frac{\bar{u}^{\varepsilon} \bar{u}_{x}^{\varepsilon}}{\bar{v}^{\varepsilon}}\right)+q_{11}-q_{12} \\
\bar{\Phi}(x, 0)=0
\end{array}\right.
$$

This system can be written as

$$
\left\{\begin{array}{l}
\bar{\Phi}_{t}-\bar{\Psi}_{x}=0 \\
\bar{\Psi}_{t}+\bar{p}_{v} \bar{\Phi}_{x}+\bar{p}_{\theta}\left(\bar{W}_{x}+\frac{\gamma-1}{R}\left(\overline{u_{x}^{\varepsilon}} \bar{\Psi}-\frac{1}{2} \bar{\Psi}_{x}^{2}\right)\right)=\varepsilon\left(\frac{u_{x}^{\varepsilon}}{v^{\varepsilon}}-\frac{\bar{u}_{x}^{\varepsilon}}{\bar{v}^{\varepsilon}}\right)-Q_{1}+q_{5}-q_{6} \\
\quad \frac{R}{\gamma-1} \bar{W}_{t}+\overline{u_{t}^{\varepsilon}} \bar{\Psi}+\bar{p} \bar{\Psi}_{x} \\
=\kappa\left(\frac{\theta_{x}^{\varepsilon}}{v^{\varepsilon}}-\frac{\overline{\theta_{x}^{\varepsilon}}}{\bar{v}^{\varepsilon}}\right)+\varepsilon \frac{u_{x}^{\varepsilon}}{v^{\varepsilon}} \bar{\Psi}_{x}+\bar{u}^{\varepsilon} Q_{1}-Q_{2}-\bar{u}^{\varepsilon}\left(q_{5}-q_{6}\right)+q_{11}-q_{12} \\
\bar{\Phi}(x, 0)=\bar{\Psi}(x, 0)=\bar{W}(x, 0)=0
\end{array}\right.
$$

By making the following rescalings,

$$
(\bar{\Phi}, \bar{\Psi}, \bar{W})(x, t)=\varepsilon(\Phi, \Psi, W)(y, \tau), \quad y=\frac{x-s(t)}{\varepsilon}, \quad \tau=\frac{t}{\varepsilon},
$$


we transform (3.5) into

$$
\left\{\begin{array}{l}
\Phi_{\tau}-\dot{s}(\varepsilon \tau) \Phi_{y}-\Psi_{y}=0 \\
\Psi_{\tau}-\dot{s}(\varepsilon \tau) \Psi_{y}-\bar{\varrho} \Phi_{y}+\overline{p_{\theta}}\left\{W_{y}+\frac{\gamma-1}{R}\left(\bar{u}_{y}^{\varepsilon} \Psi-\frac{1}{2} \Psi_{y}^{2}\right)\right\} \\
=\frac{\Psi_{y y}}{\overline{v^{\varepsilon}}}-\frac{1}{\bar{v}^{\varepsilon} v^{\varepsilon}} \Phi_{y} \Psi_{y y}+\frac{\overline{u_{y}^{\varepsilon}}}{\bar{v}^{2} v^{\varepsilon}} \Phi_{y}^{2}-Q_{1}+q_{5}-q_{6} \\
\frac{R}{\gamma-1}\left(W_{\tau}-\dot{s}(\varepsilon \tau) W_{y}\right)+\left(\overline{u_{\tau}^{\varepsilon}}-\dot{s}(\varepsilon \tau) \overline{u_{y}^{\varepsilon}}\right) \Psi+\bar{p} \Psi_{y} \\
=\frac{W_{y y}}{\overline{v^{\varepsilon}}}-\frac{1}{\bar{v}^{\varepsilon} v^{\varepsilon}} \Phi_{y} W_{y y}+\frac{\gamma-1}{R v^{\varepsilon}}\left(\overline{u_{y}^{\varepsilon}} \Psi-\frac{1}{2} \Psi_{y}^{2}\right)_{y}-\frac{\overline{\theta_{y}^{\varepsilon}}}{\bar{v}^{\varepsilon} v^{\varepsilon}} \Phi_{y}+\frac{1}{v^{\varepsilon}} \Psi_{y} \Psi_{y y}+\frac{\bar{u}_{y}^{\varepsilon}}{v^{\varepsilon}} \Psi_{y} \\
\quad+\bar{u}^{\varepsilon} Q_{1}-Q_{2}-\overline{u^{\varepsilon}}\left(q_{5}-q_{6}\right)+q_{11}-q_{12}, \\
\Phi(y, 0)=\Psi(y, 0)=W(y, 0)=0
\end{array}\right.
$$

where $\bar{\varrho}=-\left(\overline{p_{v}}+\bar{u}_{y}^{\varepsilon} / \bar{v}^{\varepsilon}\right)$, and

$$
\begin{aligned}
& Q_{1}=p\left(\bar{v}^{\varepsilon}+\Phi_{y}, \overline{\theta^{\varepsilon}}+W_{y}+\frac{\gamma-1}{R}\left(\bar{u}_{y}^{\varepsilon} \Psi-\frac{1}{2} \Psi_{y}^{2}\right)\right)-p\left(\bar{v}^{\varepsilon}, \overline{\theta^{\varepsilon}}\right) \\
& -\left(\overline{p_{v}} \Phi_{y}+\overline{p_{\theta}}\left(W_{y}+\frac{\gamma-1}{R}\left(\overline{u_{y}^{\varepsilon}} \Psi-\frac{1}{2} \Psi_{y}^{2}\right)\right)\right) \\
& \text { satisfies }\left|Q_{1}\right| \leq O(1)\left(\Phi_{y}^{2}+W_{y}^{2}+(\gamma-1) \bar{u}_{y}^{\varepsilon^{2}} \Psi^{2}+\Psi_{y}^{4}\right), \\
& Q_{2}=p\left(\overline{v^{\varepsilon}}+\Phi_{y}, \overline{\theta^{\varepsilon}}+W_{y}+\frac{\gamma-1}{R}\left(\bar{u}_{y}^{\varepsilon} \Psi-\frac{1}{2} \Psi_{y}^{2}\right)\right)\left(\bar{u}^{\varepsilon}+\Psi_{y}\right)-\bar{p} \bar{u}^{\varepsilon} \\
& -\left(\bar{u}^{\varepsilon} \overline{p_{v}} \Phi_{y}+\bar{p} \Psi_{y}+\overline{u^{\varepsilon}} \overline{p_{\theta}}\left(W_{y}+\frac{\gamma-1}{R}\left(\bar{u}_{y}^{\varepsilon} \Psi-\frac{1}{2} \Psi_{y}^{2}\right)\right)\right) \\
& \text { satisfies }\left|Q_{2}\right| \leq O(1)\left(\Phi_{y}^{2}+\Psi_{y}^{2}+W_{y}^{2}+(\gamma-1) \bar{u}_{y}^{\varepsilon^{2}} \Psi^{2}+\Psi_{y}^{4}\right) \text {. }
\end{aligned}
$$

Then we only need to show that for suitably small $\varepsilon,(3.7)$ has a unique "small" smooth solution up to $T / \varepsilon$. By the standard existence and uniqueness theory, and the continuous induction argument for hyperbolic-parabolic equations [5], it suffices to close the following a priori estimate

$$
N(\tau) \equiv\left\|\left(\Phi, \Psi, \frac{W}{\sqrt{\gamma-1}}\right)(\cdot, \tau)\right\|_{3} \leq \delta
$$

where $\delta$ is a positive small constant depending on the initial data and the strength of the shock. In fact, we have the following result.

Proposition 3.1. Suppose that the Cauchy problem (3.7) has a solution $(\Phi, \Psi, W)$

$\in C^{1}\left(\left[0, \tau_{0}\right]: H^{3}\left(R^{1}\right)\right)$ for some $\tau_{0} \in(0, T / \varepsilon]$. Then there exist positive constants $\mu_{1}, \varepsilon_{1}$ and $C$, which are independent of $\varepsilon$ and $\tau_{0}$, such that if $0<\varepsilon<\varepsilon_{1}$ and $\delta+(\gamma-1) \mu \leq \mu_{1}$, then

$$
\sup _{0 \leq \tau \leq \tau_{0}} N(\tau)^{2}+\int_{0}^{\tau_{0}}\left(\left\|\Phi_{y}(\cdot, \tau)\right\|_{2}^{2}+\left\|\left(\Psi_{y}, W_{y}\right)(\cdot, \tau)\right\|_{3}^{2}\right) d \tau \leq C \varepsilon^{(2 n+1) \nu-4}
$$

where $\nu$ is defined in Section 2.4.

The proof of Proposition 3.1 occupies the rest of this section. We separate it into two parts. In what follows, we use $c$ to denote any positive constant which is 
independent of $\varepsilon, y$ and $\tau$; and $\bar{c}$ to denote any positive constant which is independent of $\varepsilon$ and $(\gamma-1) \mu$. And we set $\varepsilon \leq 1$.

Lemma 3.2. Suppose that the conditions in Proposition 3.1 are satisfied. Then

$$
\begin{aligned}
& \left\|\left(\Phi, \Psi, \frac{W}{\sqrt{\gamma-1}}\right)(\cdot, \tau)\right\|_{1}^{2}+\int_{0}^{\tau}\left(\left\|\Phi_{y}(\cdot, \tau)\right\|^{2}+\left\|\left(\Psi_{y}, W_{y}\right)\right\|_{1}^{2}\right) d \tau \\
& +\int_{0}^{\tau} \int m V_{y}\left(\Psi^{2}+\frac{W^{2}}{\gamma-1}\right) d y d \tau \leq c \varepsilon^{(2 n+1) \nu-4},
\end{aligned}
$$

for all $\tau \in\left[0, \tau_{0}\right]$, where the constant $c$ is independent of $\tau_{0}$ and $\varepsilon$.

Proof. Step 1 Multiplying $(3.7)_{1},(3.7)_{2}$ and $(3.7)_{3}$ by $\Phi, \frac{\Psi}{\bar{\varrho}}$ and $\frac{\overline{p_{\theta}} W}{\bar{\varrho} \bar{p}}$, respectively, then integrating over $R^{1}$, and adding the resulting equations, we obtain after integration by parts that

$$
\begin{aligned}
& \frac{1}{2} \frac{d}{d \tau} \int\left(\Phi^{2}+\frac{1}{\bar{\varrho}} \Psi^{2}+\frac{R \overline{p_{\theta}}}{(\gamma-1) \bar{\varrho} \bar{p}} W^{2}\right)(y, \tau) d y \\
& +\int\left\{\left(\frac{\dot{s}}{2}\left(\frac{1}{\bar{\varrho}}\right)_{y}+\frac{(\gamma-1) \overline{p_{\theta}} \overline{u_{y}^{\varepsilon}}}{R \bar{\varrho}}\right) \Psi^{2}+\left(\frac{1}{v^{\varepsilon} \bar{\varrho}}\right)_{y} \Psi \Psi_{y}+\frac{1}{\bar{v}^{\varepsilon} \bar{\varrho}} \Psi_{y}^{2}\right\} d y \\
& +\int\left\{\frac{\dot{s} R}{2(\gamma-1)}\left(\frac{\overline{\bar{\theta}_{\theta}}}{\bar{\varrho} \overline{\bar{p}}}\right)_{y} W^{2}+\frac{\overline{p_{\theta}}}{\overline{v^{\varepsilon}} \bar{\varrho} \bar{p}} W_{y}^{2}\right\} d y \\
& =\int\left\{\frac{1}{2}\left(\frac{1}{\varrho}\right)_{\tau} \Psi^{2}-\frac{\overline{p_{\theta}}}{\bar{\varrho} \bar{p}} \bar{u}_{\tau}^{\varepsilon} \Psi W+\frac{R}{2(\gamma-1)}\left(\frac{\overline{p_{\theta}}}{\bar{\varrho} \bar{p}}\right)_{\tau} W^{2}\right\} d y \\
& +\int\left\{\left(\frac{\overline{p_{\theta}}}{\bar{\varrho}}\right)_{y}+\frac{\dot{s} \overline{p_{\theta}} \overline{u_{y}^{\varepsilon}}}{\bar{\varrho} \bar{p}}\right\} \Psi W d y-\int \frac{\gamma-1}{R}\left(\frac{\overline{p_{\theta}}}{v^{\varepsilon} \bar{\varrho} \bar{p}}\right)_{y} \overline{u_{y}^{\varepsilon}} \Psi W d y
\end{aligned}
$$

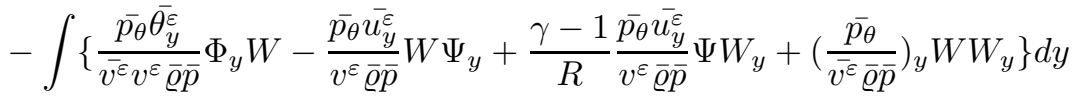

$$
\begin{aligned}
& +\int\left\{\left(\frac{\overline{u_{y}^{\varepsilon}}}{\bar{v}^{\varepsilon} v^{\varepsilon} \bar{\varrho}} \Phi_{y}^{2} \Psi+\frac{\gamma-1}{2 R}\left(\frac{\overline{p_{\theta}}}{\bar{\varrho}} \Psi+\frac{\overline{p_{\theta}}}{v^{\varepsilon} \bar{\varrho} \bar{p}} W_{y}+\left(\frac{\overline{p_{\theta}}}{v^{\varepsilon} \bar{\varrho} \bar{p}}\right)_{y} W\right) \Psi_{y}^{2}\right\} d y\right. \\
& -\int\left\{\frac{1}{\overline{v^{\varepsilon} v^{\varepsilon} \bar{\varrho}}} \Phi_{y} \Psi \Psi_{y y}-\frac{\overline{p_{\theta}}}{v^{\varepsilon} \bar{\varrho} \bar{p}} \Psi_{y} \Psi_{y y} W+\frac{\overline{p_{\theta}}}{\bar{v}^{\varepsilon} v^{\varepsilon} \bar{\varrho} \bar{p}} \Phi_{y} W W_{y y}\right\} d y \\
& -\int\left\{\frac{1}{\bar{\varrho}} Q_{1} \Psi-\frac{\overline{p_{\theta}}}{\bar{\varrho} \bar{p}}\left(\overline{u^{\varepsilon}} Q_{1}-Q_{2}\right) W\right\} d y \\
& +\int\left\{\frac{1}{\bar{\varrho}}\left(q_{5}-q_{6}\right) \Psi-\frac{\overline{p_{\theta}}}{\bar{\varrho} \bar{p}}\left(\bar{u} \varepsilon\left(q_{5}-q_{6}\right)-\left(q_{11}-q_{12}\right)\right) W\right\} d y .
\end{aligned}
$$

We denote the last two terms on the left by $I_{1}$ and $I_{2}$ respectively, and the terms on the right hand side above in order by $J_{i}, 1 \leq i \leq 8$. Now we estimate them separately as follows.

First, Using Lemma 2.3, we have $\overline{v^{\varepsilon}} \bar{\varrho}=\bar{p}-\frac{\bar{u}_{y}^{\varepsilon}}{\bar{v}^{\varepsilon}}=\bar{p}-\frac{m U_{y}}{\bar{v}^{\varepsilon}}+O(1) \varepsilon>0$ for sufficiently small $\varepsilon$. Then it follows from Young's inequality that

$$
I_{1} \geq\left(1-\eta_{1}\right) \int \frac{1}{\overline{v^{\varepsilon}} \bar{\varrho}} \Psi_{y}^{2} d y+\int\left\{\frac{\dot{s}}{2}\left(\frac{1}{\bar{\varrho}}\right)_{y}+\frac{(\gamma-1) \overline{p_{\theta}} \overline{u_{y}^{\varepsilon}}}{R \varrho \underline{\varrho}}-\frac{1}{4 \eta_{1}} \overline{v^{\varepsilon}} \bar{\varrho}\left(\left(\frac{1}{\overline{v^{\varepsilon}}}\right)_{y}\right)^{2}\right\} \Psi^{2} d y
$$

for some $\eta_{1} \in(0,1)$. Denote the second term by $\int z\left(S^{\varepsilon}\right) \Psi^{2} d y$. Then Due to (2.15), Lemma 2.3 and the fact

$$
\left|\partial_{y}(V, U, \Theta)\right|=O(1) \varepsilon, \text { on }|y| \geq \varepsilon^{\gamma-1},
$$


we get

$$
\begin{aligned}
& z\left(S^{\varepsilon}\right)=\frac{\dot{s}}{2}\left(\frac{1}{\bar{\varrho}}\right)_{y}+\frac{(\gamma-1) \overline{p_{\theta}} \overline{u_{y}^{\varepsilon}}}{R \bar{\varrho}}-\frac{1}{4 \eta_{1}} \overline{v^{\varepsilon}} \bar{\varrho}\left(\left(\frac{1}{\overline{v^{\varepsilon}} \bar{\varrho}}\right)_{y}\right)^{2} \\
& =-\frac{\dot{s}}{2}\left(\frac{\overline{v^{\varepsilon}}}{\overline{u_{y}^{\varepsilon}} / \overline{v^{\varepsilon}}-\bar{p}}\right)_{y}-\frac{(\gamma-1) \overline{u_{y}^{\varepsilon}}}{\overline{u_{y}^{\varepsilon} / \bar{v}^{\varepsilon}-\bar{p}}}+\frac{1}{4 \eta_{1}}\left(\overline{u_{y}^{\varepsilon}} / \overline{v^{\varepsilon}}-\bar{p}\right)\left(\left(\frac{1}{\overline{u_{y}^{\varepsilon}} / \overline{v^{\varepsilon}}-\bar{p}}\right)_{y}\right)^{2} \\
& =-\frac{\dot{s}}{2} m\left(\frac{V}{U_{y} / V-P}\right)_{y}-\frac{(\gamma-1) m U_{y}}{U_{y} / V-P}+\frac{1}{4 \eta_{1}} m\left(U_{y} / V-P\right)\left(\left(\frac{1}{U_{y} / V-P}\right)_{y}\right)^{2} \\
& +O(1) \varepsilon^{\gamma} m V_{y}+O(1) \varepsilon \\
& =m\left\{\frac{\dot{s}}{2}\left(\frac{V}{b_{1}-\dot{s}^{2} V}\right)_{y}-\frac{(\gamma-1) \dot{s} V_{y}}{b_{1}-\dot{s}^{2} V}-\frac{1}{4 \eta_{1}}\left(b_{1}-\dot{s}^{2} V\right)\left(\left(\frac{1}{b_{1}-\dot{s}^{2} V}\right)_{y}\right)^{2}\right\} \\
& +O(1) \varepsilon^{\gamma} m V_{y}+O(1) \varepsilon \\
& =m V_{y} \frac{\dot{s}}{4\left(b_{1}-\dot{s}^{2} V\right)^{2}}\left\{2 b_{1}-4(\gamma-1)\left(b_{1}-\dot{s}^{2} V\right)-\frac{1}{\eta_{1}} \frac{\dot{s}^{3} V_{y}}{b_{1}-\dot{s}^{2} V}\right\} \\
& +O(1) \varepsilon^{\gamma} m V_{y}+O(1) \varepsilon \\
& =m V_{y} \frac{\dot{s}}{4\left(b_{1}-\dot{s}^{2} V\right)^{2}}\left\{\left[b_{1}+\frac{\dot{s}^{2} R \Theta}{b_{1}-\dot{s}^{2} V}+\left(b_{1}-\dot{s}^{2} V\right)-4(\gamma-1)\left(b_{1}-\dot{s}^{2} V\right)\right]\right. \\
& \left.+\left[\left(\frac{1}{\eta_{1}}-1\right)\left(\frac{\dot{s}^{2} R \Theta}{b_{1}-\dot{s}^{2} V}-\dot{s}^{2} V\right)\right]\right\}+O(1) \varepsilon^{\gamma} m V_{y}+O(1) \varepsilon \\
& \equiv: m V_{y} \frac{\dot{s}}{4\left(b_{1}-\dot{s}^{2} V\right)^{2}}\left\{z_{1}(H)+z_{2}(H)\right\}+O(1) \varepsilon^{\gamma} m V_{y}+O(1) \varepsilon \text {. }
\end{aligned}
$$

As in [16], using the fact

$$
\dot{s}^{2} \geq \frac{\gamma p_{l}}{v_{r}}-c(\gamma-1)\left(v_{r}-v_{l}\right) \geq \frac{\gamma p_{l}}{v_{r}}-\bar{c}(\gamma-1) \mu
$$

which follows from the Rankine-Hugniot condition, we can obtain

$$
z_{1}(H) \geq\left(p_{l}+\dot{s}^{2} v_{l}\right)+\frac{\dot{s}^{2} R \theta_{r}}{p_{l}}-4(\gamma-1) p_{l} \geq \frac{(5-2 \gamma) R \theta_{l}}{v_{r}}-c(\gamma-1) \mu .
$$

On the other hand,

$$
z_{2}(H) \geq\left(\frac{1}{\eta_{1}}-1\right)\left(\frac{\dot{s}^{2} R \theta_{r}}{p_{l}}-\dot{s}^{2} v_{r}\right)=-\left(\frac{1}{\eta_{1}}-1\right) \frac{\dot{s}^{4} v_{r}}{p_{l}}\left(v_{r}-v_{l}\right) \geq-(\gamma-1) \mu
$$

if we choose $\frac{\dot{s}^{4} v_{r}}{\dot{s}^{4} v_{r}+(\gamma-1) p_{l}} \leq \eta_{1}<1$. So there is a constant $\underline{c}>0$, such that

$$
I_{1} \geq \underline{c} \int m V_{y} \Psi^{2} d y+\underline{c} \int \Psi_{y}^{2} d y-\left(\bar{c}(\gamma-1) \mu+c \varepsilon^{\gamma}\right) \int m V_{y} \Psi^{2} d y-c \varepsilon\|\Psi(\cdot, \tau)\|^{2} .
$$

Next we estimate $I_{2}$. Denote the first term of $I_{2}$ as $I_{2}^{(1)}$. Then we have

$$
\begin{aligned}
I_{2}^{(1)}= & -\frac{\dot{s} R}{2(\gamma-1)} \int m\left(\frac{V}{\Theta\left(U_{y} / V-P\right)}\right)_{y} W^{2} d y \\
& +O(1) \varepsilon^{\nu} \int m V_{y} \frac{W^{2}}{\gamma-1} d y+O(1) \varepsilon\left\|\frac{W}{\sqrt{\gamma-1}}(\cdot, \tau)\right\|^{2}
\end{aligned}
$$




$$
\begin{aligned}
&= \frac{\dot{s} R}{2(\gamma-1)} \int m \frac{b_{1} \Theta V_{y}+\left(\dot{s}^{2} V-b_{l}\right) V \Theta_{y}}{\Theta^{2}\left(\dot{s}^{2} V-b_{1}\right)^{2}} W^{2} d y \\
&+O(1) \varepsilon^{\nu} \int m V_{y} \frac{W^{2}}{\gamma-1} d y+O(1) \varepsilon\left\|\frac{W}{\sqrt{\gamma-1}}(\cdot, \tau)\right\|^{2} \\
& \geq \frac{\dot{s} R}{2(\gamma-1)} \int m \frac{b_{1} \Theta V_{y}+\dot{s}^{2}\left(V-v_{l}\right) V \Theta_{y}}{\Theta^{2}\left(\dot{s}^{2} V-b_{1}\right)^{2}} W^{2} d y \\
&-c \varepsilon^{\nu} \int m V_{y} \frac{W^{2}}{\gamma-1} d y-c \varepsilon\left\|\frac{W}{\sqrt{\gamma-1}}(\cdot, \tau)\right\|^{2} \\
& \geq \int \frac{\dot{s} R b_{1}}{2(\gamma-1) \Theta\left(\dot{s}^{2} V-b_{1}\right)^{2}} m V_{y} W^{2} d y \\
&-\left(\bar{c}(\gamma-1) \mu+c \varepsilon^{\nu}\right) \int m V_{y} \frac{W^{2}}{\gamma-1} d y+c \varepsilon\left\|\frac{W}{\sqrt{\gamma-1}}(\cdot, \tau)\right\|^{2} \\
& \geq \underline{c} \int m V_{y} \frac{W^{2}}{\gamma-1} d y,
\end{aligned}
$$

for some constant $\underline{c}>0$, provided that $(\gamma-1) \mu$ and $\varepsilon$ are sufficiently small, where we have used (2.15)-(2.16) and Lemma 2.3. So

$$
I_{2} \geq \underline{c} \int m V_{y} \frac{W^{2}}{\gamma-1} d y+\underline{c} \int W_{y}^{2} d y
$$

for some constant $\underline{c}>0$.

Now we estimate the terms $J_{i}, 1 \leq i \leq 8$. First Lemma 2.3 gives

$$
J_{1} \leq c \varepsilon\left(\|\Psi(\cdot, \tau)\|^{2}+\left\|\frac{W}{\sqrt{\gamma-1}}(\cdot, \tau)\right\|^{2}\right) .
$$

and

$$
\begin{aligned}
J_{2} \leq & \int m\left|R\left(\frac{1}{U_{y} / V-P}\right)_{y}+\frac{\dot{s} V U_{y}}{\Theta\left(U_{y} / V-P\right)}\|\Psi\| W\right| d y \\
& +c \varepsilon^{\nu} \int m V_{y}\left(\Psi^{2}+W^{2}\right) d y+c \varepsilon\left(\|\Psi(\cdot, \tau)\|^{2}+\|W(\cdot, \tau)\|^{2}\right) \\
= & \int \frac{m \dot{s}^{2} V V_{y}}{\Theta\left(\dot{s}^{2} V-b_{1}\right)^{2}}\left|\left(P-p_{l}\right)+\dot{s}^{2}\left(V-v_{l}\right)\|\Psi|\| W| d y\right. \\
& +c \varepsilon^{\nu} \int m V_{y}\left(\Psi^{2}+W^{2}\right) d y+c \varepsilon\left(\|\Psi(\cdot, \tau)\|^{2}+\|W(\cdot, \tau)\|^{2}\right) \\
\leq & \left(\bar{c} \eta^{-1}(\gamma-1) \mu+c \varepsilon^{\nu}\right) \int m V_{y} \Psi^{2} d y+\left(\eta+c \varepsilon^{\nu}\right) \int m V_{y} \frac{W^{2}}{\gamma-1} d y \\
& +c \varepsilon\left(\|\Psi(\cdot, \tau)\|^{2}+\|W(\cdot, \tau)\|^{2}\right),
\end{aligned}
$$

where $\eta>0$ is a constant to be determined later, which is different from the one in Theorem 1.2 and be temporarily used in this subsection. Using (2.15)-(2.16) and Lemma 2.3 again, one finds

$$
\left|\partial_{y}^{l} S^{\varepsilon}\right|^{2} \leq \bar{c} \mu m V_{y}+O(1) \varepsilon, 1 \leq l \leq 2 .
$$

Thus,

$J_{3}=-\frac{\gamma-1}{R} \int\left(\frac{\overline{p_{\theta}}}{\overline{v^{\varepsilon}} \bar{\varrho} \bar{p}}\right)_{y} \overline{u_{y}^{\varepsilon}} \Psi W d y-\frac{\gamma-1}{R} \int \frac{\overline{p_{\theta}}}{\overline{v^{\varepsilon} v^{\varepsilon}} \bar{\varrho} \bar{p}} \Phi_{y}\left(u_{y y}^{\bar{\varepsilon}} \Psi W+\overline{u_{y}^{\varepsilon}}\left(\Psi_{y} W+\Psi W_{y}\right)\right) d y$ 


$$
\begin{aligned}
\leq & c(\gamma-1) \int\left(\bar{v}_{y}^{\varepsilon}+\bar{u}_{y}^{\varepsilon}+u_{y y}^{\bar{\varepsilon}}{ }^{2}+\bar{\theta}_{y}^{2}\right)\left(\Psi^{2}+W^{2}\right) d y \\
& +c(\gamma-1)\left(\|\Psi\|_{L^{\infty}}+\|W\|_{L^{\infty}}\right)\left\|\Phi_{y}\right\|^{2}+c\|W\|_{L^{\infty}}\left\|\Psi_{y}\right\|^{2}+c\|\Psi\|_{L^{\infty}}\left\|W_{y}\right\|^{2} \\
\leq & \bar{c}(\gamma-1) \mu \int m V_{y}\left(\Psi^{2}+\frac{W^{2}}{\gamma-1}\right) d y+c \delta\left(\left\|\Phi_{y}(\cdot, \tau)\right\|^{2}+\left\|\Psi_{y}(\cdot, \tau)\right\|^{2}+\left\|W_{y}(\cdot, \tau)\right\|^{2}\right) \\
& +c \varepsilon\left(\|\Psi(\cdot, \tau)\|^{2}+\|W(\cdot, \tau)\|^{2}\right) .
\end{aligned}
$$

Noticing that $\bar{\varrho}>0$ and $\overline{p_{v}}<0$ for sufficiently small $\varepsilon, J_{4}$ can be estimated as

$$
\begin{aligned}
J_{4} \leq & -\eta \int \overline{p_{v}} \Phi_{y}^{2} d y+\frac{1}{4} \underline{c} \int\left(\Psi_{y}^{2}+W_{y}^{2}\right) d y \\
& \left.+c(\gamma-1) \int \overline{u_{y}^{\varepsilon}} \Psi^{2} d y+c \int\left(\eta^{-1}{\overline{\theta_{y}^{\varepsilon}}}^{2}+\bar{u}_{y}^{\varepsilon^{2}}+{\overline{v_{y}}}^{2}+u_{y y}^{\bar{\varepsilon}}{ }^{2}+{\overline{\theta_{y}^{\varepsilon}}}^{2}\right) W^{2}\right) d y \\
\leq & -\eta \int \overline{p_{v}} \Phi_{y}^{2} d y+\frac{1}{4} \underline{c} \int\left(\Psi_{y}^{2}+W_{y}^{2}\right) d y \\
& +\bar{c}(\gamma-1) \mu\left\{\int m V_{y} \Psi^{2} d y+\left(\eta^{-1}+1\right) \int m V_{y} \frac{W^{2}}{\gamma-1} d y\right\} \\
& +c \varepsilon\left(\|\Psi(\cdot, \tau)\|^{2}+\left(\eta^{-1}+1\right)\|W(\cdot, \tau)\|^{2}\right),
\end{aligned}
$$

Continuing, we compute that

$$
\begin{aligned}
J_{5} & =\int \frac{\overline{u_{y}^{\varepsilon}}}{\bar{v}^{2} v^{\varepsilon} \bar{\varrho}} \Psi \Phi_{y}^{2} d y+\frac{\gamma-1}{2 R} \int \frac{\overline{p_{\theta}}}{\bar{\varrho}} \Psi \Psi_{y}^{2} d y-\frac{\gamma-1}{R} \int \frac{\overline{p_{\theta}}}{v^{\varepsilon} \bar{\varrho} \bar{p}} W \Psi_{y} \Psi_{y y} d y \\
& \leq c\|\Psi\|_{L^{\infty}}\left\|\Phi_{y}\right\|^{2}+c\left(\|\Psi\|_{L^{\infty}}+\|W\|_{L^{\infty}}\right)\left\|\Psi_{y}\right\|^{2}+c\|W\|_{L^{\infty}}\left\|\Psi_{y y}\right\|^{2} \\
& \leq c \delta\left(\left\|\Phi_{y}\right\|^{2}+\left\|\Psi_{y}\right\|^{2}+\left\|\Psi_{y y}\right\|^{2}\right) .
\end{aligned}
$$

Similarly,

$$
\begin{aligned}
J_{6} & \leq c\left(\|\Psi\|_{L^{\infty}}+\|W\|_{L^{\infty}}\right)\left(\left\|\Phi_{y}\right\|^{2}+\left\|\Psi_{y y}\right\|^{2}\right)+c\|W\|_{L^{\infty}}\left(\left\|\Psi_{y}\right\|^{2}+\left\|W_{y y}\right\|^{2}\right) \\
& \leq c \delta\left(\left\|\Phi_{y}\right\|^{2}+\left\|\Psi_{y}\right\|^{2}+\left\|\Psi_{y y}\right\|^{2}+\left\|W_{y y}\right\|^{2}\right) .
\end{aligned}
$$

In view of (3.8), we get

$$
\begin{aligned}
J_{7} \leq & c \int\left(\Phi_{y}^{2}+(\gamma-1) \bar{u}_{y}^{\bar{\varepsilon}^{2}} \Psi^{2}+\Psi_{y}^{4}+W_{y}^{2}\right)|\Psi| d y \\
& +\left(\Phi_{y}^{2}+\Psi_{y}^{2}+(\gamma-1) \bar{u}_{y}^{\varepsilon} \Psi^{2}+\Psi_{y}^{4}+W_{y}^{2}\right)|W| d y \\
\leq & c\left(\|\Psi\|_{L^{\infty}}+\|W\|_{L^{\infty}}\right)\left\{\left\|\Phi_{y}\right\|^{2}+\left(1+\left\|\Psi_{y}\right\|_{L^{\infty}}^{2}\right)\left\|\Psi_{y}\right\|^{2}+\left\|W_{y}\right\|^{2}\right\} \\
& +c \int(\gamma-1)\left|\bar{u}_{y}^{\varepsilon}\right|^{2} \Psi^{2} d y \\
\leq & c \delta\left(\left\|\Phi_{y}\right\|^{2}+\left\|\Psi_{y}\right\|^{2}+\left\|W_{y}\right\|^{2}\right)+\bar{c}(\gamma-1) \mu \int m V_{y} \Psi^{2} d y+c \varepsilon\|\Psi(\cdot, \tau)\|^{2},
\end{aligned}
$$

provided that $\|\Psi(\cdot, \tau)\|_{2}$ is bounded. Finally, Young's inequality and Lemma 2.2 lead to

$$
\begin{aligned}
J_{8} & \leq c \varepsilon\left(\|\Psi\|^{2}+\|W\|^{2}\right)+c \varepsilon^{-1} \int\left(q_{5}^{2}+q_{6}^{2}+q_{11}^{2}+q_{12}^{2}\right) d y \\
& \leq c \varepsilon\left(\|\Psi\|^{2}+\|W\|^{2}\right)+c \varepsilon^{(2 n+1) \nu-3} .
\end{aligned}
$$


Collecting all the estimates we have obtained, we get

$$
\begin{aligned}
& \frac{1}{2} \frac{d}{d \tau} \int\left(\Phi^{2}+\frac{1}{\bar{\varrho}} \Psi^{2}+\frac{R \overline{p_{\theta}}}{(\gamma-1) \bar{\varrho} \bar{p}} W^{2}\right)(y, \tau) d y+\underline{c} \int\left(\Psi_{y}^{2}+W_{y}^{2}\right) d y \\
& \leq-\eta \int \overline{p_{v}} \Phi_{y}^{2} d y+c \delta\left\|\Phi_{y}\right\|^{2}+\frac{1}{4} \underline{c} \int\left(\Psi_{y}^{2}+W_{y}^{2}\right) d y+c \delta\left(\left\|\Psi_{y}\right\|^{2}+\left\|W_{y}\right\|^{2}\right) \\
& \quad+\left(-\underline{c}+\bar{c}\left(\eta^{-1}+1\right)(\gamma-1) \mu+c \varepsilon^{\nu}\right) \int m V_{y}\left(\Psi^{2}+\frac{W^{2}}{\gamma-1}\right) d y+\eta \int m V_{y} \frac{W^{2}}{\gamma-1} d y \\
& \quad+c \delta\left(\left\|\Psi_{y y}\right\|^{2}+\left\|W_{y y}\right\|^{2}\right)+c\left(\eta^{-1}+1\right) \varepsilon\left(\|\Psi(\cdot, \tau)\|^{2}+\left\|\frac{W}{\sqrt{\gamma-1}}(\cdot, \tau)\right\|^{2}\right) \\
& \quad+c \varepsilon^{(2 n+1) \nu-3} .
\end{aligned}
$$

By choosing $\delta$ sufficiently small, we conclude that

$$
\begin{aligned}
& \frac{d}{d \tau} \int\left(\Phi^{2}+\frac{1}{\bar{\varrho}} \Psi^{2}+\frac{R \overline{p_{\theta}}}{(\gamma-1) \bar{\varrho} \bar{p}} W^{2}\right)(y, \tau) d y+\underline{c} \int\left(\Psi_{y}^{2}+W_{y}^{2}\right) d y \\
& +\eta \int \overline{p_{v}} \Phi_{y}^{2} d y+2 \underline{c} \int m V_{y}\left(\Psi^{2}+\frac{W^{2}}{\gamma-1}\right) d y \\
& \leq\left(\bar{c}\left(\eta^{-1}+1\right)(\gamma-1) \mu+c \varepsilon^{\nu}\right) \int m V_{y}\left(\Psi^{2}+\frac{W^{2}}{\gamma-1}\right) d y+\eta \int m V_{y} \frac{W^{2}}{\gamma-1} d y \\
& \quad+c \delta\left(\left\|\Psi_{y y}\right\|^{2}+\left\|W_{y y}\right\|^{2}\right)+c\left(\eta^{-1}+1\right) \varepsilon\left(\|\Psi(\cdot, \tau)\|^{2}+\left\|\frac{W}{\sqrt{\gamma-1}}(\cdot, \tau)\right\|^{2}\right) \\
& \quad+c \varepsilon^{(2 n+1) \nu-3} .
\end{aligned}
$$

Step 2 We first rewrite (3.7) as

$$
\left\{\begin{array}{l}
\Phi_{\tau}-\dot{s}(\varepsilon \tau) \Phi_{y}-\Psi_{y}=0, \\
\Psi_{\tau}-\dot{s}(\varepsilon \tau) \Psi_{y}+p\left(v^{\varepsilon}, \theta^{\varepsilon}\right)-p\left(\overline{v^{\varepsilon}}, \bar{\theta}^{\varepsilon}\right) \\
=\frac{\Psi_{y y}}{\bar{v}^{\varepsilon}}-\frac{1}{\bar{v}^{\varepsilon} v^{\varepsilon}} \Phi_{y} \Psi_{y y}-\frac{\bar{u}_{y}^{\varepsilon}}{\bar{v}^{\varepsilon} v^{\varepsilon}} \Phi_{y}+q_{5}-q_{6} \\
\frac{R}{\gamma-1}\left(W_{\tau}-\dot{s}(\varepsilon \tau) W_{y}\right)+\left(\overline{u_{\tau}^{\varepsilon}}-\dot{s}(\varepsilon \tau) \overline{u_{y}^{\varepsilon}}\right) \Psi+p\left(v^{\varepsilon}, \theta^{\varepsilon}\right) \Psi_{y} \\
=\frac{W_{y y}}{\bar{v}^{\varepsilon}}-\frac{1}{\bar{v}^{\varepsilon} v^{\varepsilon}} \Phi_{y} W_{y y}+\frac{\gamma-1}{R v^{\varepsilon}}\left(\overline{u_{y}^{\varepsilon}} \Psi-\frac{1}{2} \Psi_{y}^{2}\right)_{y}-\frac{\overline{\theta^{\varepsilon}}}{\bar{v}^{\varepsilon} v^{\varepsilon}} \Phi_{y}+\frac{1}{v^{\varepsilon}} \Psi_{y} \Psi_{y y}+\frac{\bar{u}_{y}^{\varepsilon}}{v^{\varepsilon}} \Psi_{y} \\
-\bar{u}^{\varepsilon}\left(q_{5}-q_{6}\right)+q_{11}-q_{12}, \\
\Phi(y, 0)=\Psi(y, 0)=W(y, 0)=0 .
\end{array}\right.
$$

Then differentiating (3.17) $)_{2,3}$ with respect to $y$, multiplying both sides of the resulting equations by $\Psi_{y}, W_{y}$, respectively, then summing them up, and integrating over $R^{1}$, we obtain after integration by parts and using Young's inequality that

$$
\begin{aligned}
& \frac{1}{2} \frac{d}{d \tau}\left(\left\|\Psi_{y}\right\|^{2}+\frac{R}{\gamma-1}\left\|W_{y}\right\|^{2}\right)+\int \frac{1}{\bar{v}^{\varepsilon}}\left(\Psi_{y y}^{2}+W_{y y}^{2}\right) d y \\
& =\int \frac{\Phi_{y}}{\overline{v^{\varepsilon}} v^{\varepsilon}}\left(\Psi_{y y}^{2}+W_{y y}^{2}\right) d y \\
& \quad+\int \Psi_{y y}\left\{p\left(v^{\varepsilon}, \theta^{\varepsilon}\right)-p\left(\bar{v}^{\varepsilon}, \overline{\theta^{\varepsilon}}\right)+\frac{\overline{u_{y}^{\varepsilon}}}{\bar{v}^{\varepsilon} v^{\varepsilon}} \Phi_{y}-q_{5}+q_{6}\right\} d y \\
& \quad+\int W_{y y}\left\{\left(\bar{u}_{\tau}^{\varepsilon}-\dot{s}(\varepsilon \tau) \overline{u_{y}^{\varepsilon}}\right) \Psi+p\left(v^{\varepsilon}, \theta^{\varepsilon}\right) \Psi_{y}-\frac{\gamma-1}{R v^{\varepsilon}}\left(u_{y y}^{\bar{\varepsilon}} \Psi+\bar{u}_{y}^{\varepsilon} \Psi_{y}-\Psi_{y} \Psi_{y y}\right)\right.
\end{aligned}
$$




$$
\begin{aligned}
& \left.+\frac{\overline{\theta_{y}^{\varepsilon}}}{\bar{v}^{\varepsilon} v^{\varepsilon}} \Phi_{y}-\frac{1}{v^{\varepsilon}} \Psi_{y} \Psi_{y y}-\frac{\overline{u_{y}^{\varepsilon}}}{v^{\varepsilon}} \Psi_{y}+\bar{u}^{\varepsilon}\left(q_{5}-q_{6}\right)-\left(q_{11}-q_{12}\right)\right\} d y \\
\leq & c\left(\left\|\Phi_{y}\right\|_{L^{\infty}}+\left\|\Psi_{y}\right\|_{L^{\infty}}\right) \int \frac{1}{\bar{v}^{\varepsilon}}\left(\Psi_{y y}^{2}+W_{y y}^{2}\right) d y+\frac{1}{4} \int \frac{1}{\bar{v}^{\varepsilon}}\left(\Psi_{y y}^{2}+W_{y y}^{2}\right) d y \\
& +c\left\{\left\|\Phi_{y}\right\|^{2}+\left(1+\left\|\Psi_{y}\right\|_{L^{\infty}}^{2}\right)\left\|\Psi_{y}\right\|^{2}+\left\|W_{y}\right\|^{2}\right\}+c \varepsilon\|\Psi(\cdot, \tau)\|^{2} \\
& +c \int\left(\bar{u}_{y}^{\varepsilon}+u_{y y}^{\bar{\varepsilon}}{ }^{2}\right) \Psi^{2} d y+\int\left(q_{5}^{2}+q_{6}^{2}+q_{11}^{2}+q_{12}^{2}\right) d y \\
\leq & \left(c \delta+\frac{1}{4}\right) \int \frac{1}{\overline{v^{\varepsilon}}}\left(\Psi_{y y}^{2}+W_{y y}^{2}\right) d y+c\left(\left\|\Phi_{y}\right\|^{2}+\int m V_{y} \Psi^{2} d y+\left\|\Psi_{y}\right\|^{2}+\left\|W_{y}\right\|^{2}\right) \\
& +c \varepsilon\|\Psi(\cdot, \tau)\|^{2}+c \varepsilon^{(2 n+1) \nu-3},
\end{aligned}
$$

provided that $\left\|\left(\Phi_{y}, \Psi_{y}, W_{y}\right)\right\|_{L^{\infty}}$ is bounded, where we have used (3.1), (3.3), (3.6), (3.15) and (2.60). By taking $\delta$ sufficiently small, we arrive at

$$
\begin{aligned}
& \frac{d}{d \tau}\left(\left\|\Psi_{y}\right\|^{2}+\frac{R}{\gamma-1}\left\|W_{y}\right\|^{2}\right)+\int \frac{1}{\bar{v}^{\varepsilon}}\left(\Psi_{y y}^{2}+W_{y y}^{2}\right) d y \\
& -c\left(\left\|\Phi_{y}\right\|^{2}+\int m V_{y} \Psi^{2} d y+\left\|\Psi_{y}\right\|^{2}+\left\|W_{y}\right\|^{2}\right) \\
& \leq c \varepsilon\|\Psi(\cdot, \tau)\|^{2}+c \varepsilon^{(2 n+1) \nu-3} .
\end{aligned}
$$

We denote the constant $c$ on the left by $c_{1}$.

Step 3 Noting that $\frac{d}{d \tau}\left\|\Phi_{y}\right\|^{2}$ is not included in (3.18), we need to estimate it separately. Multiply both sides of $(3.7)_{2}$ by $\Phi_{y}$ and integrate over $R^{1}$ to obtain

$$
\begin{aligned}
& \int\left\{\Phi_{y} \Psi_{\tau}-\dot{s} \Phi_{y} \Psi_{y}+\overline{p_{v}} \Phi_{y}^{2}+\overline{p_{\theta}} \Phi_{y}\left(W_{y}+\frac{\gamma-1}{R}\left(\overline{u_{y}^{\varepsilon}} \Psi-\frac{1}{2} \Psi_{y}^{2}\right)\right)\right\} d y \\
& =\int \frac{\Phi_{y} \Psi_{y y}}{\bar{v}^{\varepsilon}} d y-\int \frac{1}{\bar{v}^{\varepsilon} v^{\varepsilon}} \Phi_{y}^{2} \Psi_{y y} d y-\int \frac{\overline{u_{y}^{\varepsilon}}}{\bar{v}^{\varepsilon} v^{\varepsilon}} \Phi_{y}^{2} d y+\int \Phi_{y}\left(-Q_{1}+q_{5}-q_{6}\right) d y .
\end{aligned}
$$

The first term on the left can be written as

$$
\begin{aligned}
\int \Phi_{y} \Psi_{\tau} d y & =\frac{d}{d \tau} \int \Phi_{y} \Psi d y-\Phi_{y \tau} \Psi d y \\
& =-\frac{d}{d \tau} \int \Phi \Psi_{y} d y+\int \Psi_{y}^{2} d y+\dot{s} \int \Phi_{y} \Psi_{y} d y
\end{aligned}
$$

and the first term on the right reads

$$
\begin{aligned}
\int \frac{\Phi_{y} \Psi_{y y}}{\bar{v}^{\varepsilon}} d y & =\int \frac{1}{\bar{v}^{\varepsilon}} \Phi_{y}\left(\Phi_{y \tau}-\dot{s} \Phi_{y y}\right) d y \\
& =\frac{1}{2} \frac{d}{d \tau} \int \frac{\Phi_{y}^{2}}{\bar{v}^{\varepsilon}} d y+\frac{1}{2} \int \frac{\Phi_{y}^{2}}{\bar{v}^{2}}\left(\overline{v_{\tau}^{\varepsilon}}-\dot{s} \bar{v}_{y}^{\varepsilon}\right) d y \\
& =\frac{1}{2} \frac{d}{d \tau} \int \frac{\Phi_{y}^{2}}{\bar{v}^{\varepsilon}} d y+\frac{1}{2} \int \frac{\bar{u}_{y}^{\varepsilon}}{\bar{v}^{2}} \Phi_{y}^{2} d y
\end{aligned}
$$

where we have used $(3.7)_{1}$ and $(2.49)_{1}$. Substituting them into (3.19), we get

$$
\frac{d}{d \tau} \int\left(\frac{1}{2} \frac{\Phi_{y}^{2}}{\bar{v}^{\varepsilon}}+\Phi \Psi_{y}\right) d y=\int \overline{p_{v}} \Phi_{y}^{2} d y+\int \overline{p_{\theta}} \Phi_{y}\left(W_{y}+\frac{\gamma-1}{R}\left(\overline{u_{y}^{\varepsilon}} \Psi-\frac{1}{2} \Psi_{y}^{2}\right)\right) d y
$$




$$
\begin{aligned}
& +\int \Psi_{y}^{2} d y+\int \frac{1}{\overline{v^{\varepsilon}} v^{\varepsilon}} \Phi_{y}^{2} \Psi_{y y} d y+\int\left(\frac{1}{\overline{v^{\varepsilon}} v^{\varepsilon}}-\frac{1}{2 \bar{v}^{2}}\right) u_{y}^{\bar{\varepsilon}} \Phi_{y}^{2} d y \\
& +\int \Phi_{y} Q_{1} d y-\int \Phi_{y}\left(q_{5}-q_{6}\right) d y .
\end{aligned}
$$

We denote the second term on the right by $\omega$. By Young's inequality, one finds

$$
\begin{aligned}
\omega & \leq-\frac{1}{8} \int \overline{p_{v}} \Phi_{y}^{2} d y+c\left(\left\|W_{y}\right\|^{2}+(\gamma-1) \int \overline{u_{y}^{\varepsilon}} \Psi^{2} d y+\left\|\Psi_{y}\right\|_{L^{\infty}}^{2}\left\|\Psi_{y}\right\|^{2}\right) \\
& \leq-\frac{1}{8} \int \overline{p_{v}} \Phi_{y}^{2} d y+c\left(\left\|\Psi_{y}\right\|^{2}+\left\|W_{y}\right\|^{2}\right)+\bar{c}(\gamma-1) \mu \int m V_{y} \Psi^{2} d y+c \varepsilon\|\Psi(\cdot, \tau)\|^{2},
\end{aligned}
$$

provided that $\left\|\Psi_{y}\right\|_{L^{\infty}}$ is bounded. Similarly,

$$
\begin{aligned}
\int \frac{1}{\overline{v^{\varepsilon}} v^{\varepsilon}} \Phi_{y}^{2} \Psi_{y y} d y & \leq-\frac{1}{8} \int \overline{p_{v}} \Phi_{y}^{2} d y+c\left\|\Phi_{y}\right\|_{L^{\infty}}^{2}\left\|\Psi_{y y}\right\|^{2} \\
& \leq-\frac{1}{8} \int \overline{p_{v}} \Phi_{y}^{2} d y+c \delta\left\|\Psi_{y y}\right\|^{2}
\end{aligned}
$$

Noting that $\bar{u}_{y}^{\varepsilon}=m U_{y}+O(1) \varepsilon$ and $U_{y}<0$, we get

$$
\int\left(\frac{1}{\bar{v}^{\varepsilon} v^{\varepsilon}}-\frac{1}{2 \bar{v}^{\varepsilon^{2}}}\right) \bar{u}_{y}^{\varepsilon} \Phi_{y}^{2} d y=\int \frac{\overline{u_{y}^{\varepsilon}}}{2 \bar{v}^{2}} \Phi_{y}^{2} d y-\int \frac{\Phi_{y}^{3}}{\bar{v}^{\varepsilon^{2}} v^{\varepsilon}} d y \leq C(\varepsilon+\delta)\left\|\Phi_{y}\right\|^{2} .
$$

Due to $(3.8)$

$$
\begin{aligned}
\int \Phi_{y} Q_{1} d y & \leq c \int\left|\Phi_{y}\right|\left(\Phi_{y}^{2}+(\gamma-1) \bar{u}_{y}^{2} \Psi^{2}+\Psi_{y}^{4}+W_{y}^{2}\right) d y \\
& \leq c \delta\left(\left\|\Phi_{y}\right\|^{2}+\left\|\Psi_{y}\right\|^{2}+\left\|W_{y}\right\|^{2}\right)+\bar{c}(\gamma-1) \mu \int m V_{y} \Psi^{2} d y+c \varepsilon\|\Psi(\cdot, \tau)\|^{2} .
\end{aligned}
$$

Lemma 2.2 and Young's inequality yield

$$
-\int \Phi_{y}\left(q_{5}-q_{6}\right) d y \leq \varepsilon\left\|\Phi_{y}\right\|^{2}+\frac{1}{4 \varepsilon} \int\left(q_{5}^{2}+q_{6}^{2}\right) d y \leq \varepsilon\left\|\Phi_{y}\right\|^{2}+c \varepsilon^{(2 n+1) \nu-3} .
$$

Collecting all the estimates we have obtained and taking $\delta$ to be sufficiently small, we have

$$
\begin{aligned}
& \frac{d}{d \tau} \int\left(\frac{\Phi_{y}^{2}}{\bar{v}^{\varepsilon}}+2 \Phi \Psi_{y}\right) d y-\int \overline{p_{v}} \Phi_{y}^{2} d y-c\left(\left\|\Psi_{y}\right\|^{2}+\left\|W_{y}\right\|^{2}\right) \\
& \leq c \varepsilon\left\|\Phi_{y}\right\|^{2}+c \delta\left\|\Psi_{y y}\right\|^{2}+\bar{c}(\gamma-1) \mu \int m V_{y} \Psi^{2} d y+c \varepsilon\|\Psi(\cdot, \tau)\|^{2}+c \varepsilon^{(2 n+1) \nu-3} .
\end{aligned}
$$

We denote the constant $c$ on the left by $c_{2}$.

Step 4 Choosing suitable constants $\beta_{1}, \beta_{2}>0$ and adding up the three inequalities (3.16), $\beta_{1}(3.18), \beta_{2}(3.20)$, we obtain the following inequality

$$
\begin{aligned}
& \frac{d}{d \tau} \int\left\{\Phi^{2}+\frac{1}{\bar{\varrho}} \Psi^{2}+\beta_{2} \frac{\Phi_{y}^{2}}{\overline{v^{\varepsilon}}}+2 \beta_{2} \Phi \Psi_{y}+\beta_{1} \Psi_{y}^{2}+\frac{R}{\gamma-1}\left(\frac{\overline{p_{\theta}}}{\bar{\varrho} \bar{p}} W^{2}+\beta_{1} W_{y}^{2}\right)\right\} d y \\
& -\int\left(\left(\beta_{2}-\eta\right) \bar{p}_{v}+c_{1} \beta_{1}\right) \Phi_{y}^{2} d y+\int\left(\underline{c}-c_{1} \beta_{1}-c_{2} \beta_{2}\right)\left(\Psi_{y}^{2}+W_{y}^{2}\right) d y
\end{aligned}
$$




$$
\begin{aligned}
+ & \beta_{1} \int \frac{1}{\overline{v^{\varepsilon}}}\left(\Psi_{y y}^{2}+W_{y y}^{2}\right) d y+\left(2 \underline{c}-c_{1} \beta_{1}\right) \int m V_{y}\left(\Psi^{2}+\frac{W^{2}}{\gamma-1}\right) d y \\
\leq & c \beta_{1} \varepsilon\left\|\Phi_{y}\right\|^{2}+c \delta\left(\beta_{2}+1\right)\left\|\Psi_{y y}\right\|^{2}+c \delta\left\|W_{y y}\right\|^{2} \\
& +\left(\bar{c}\left(\eta^{-1}+\beta_{2}+1\right)(\gamma-1) \mu+c \varepsilon^{\nu}\right) \int m V_{y}\left(\Psi^{2}+\frac{W^{2}}{\gamma-1}\right) d y+\eta \int m V_{y} \frac{W^{2}}{\gamma-1} d y \\
& +c\left(\eta^{-1}+\beta_{1}+\beta_{2}+1\right) \varepsilon\left(\|\Psi(\cdot, \tau)\|^{2}+\left\|\frac{W}{\sqrt{\gamma-1}}(\cdot, \tau)\right\|^{2}\right)+c \varepsilon^{(2 n+1) \nu-3} .
\end{aligned}
$$

To get desired signs, we first choose $\beta_{1}=2 \beta_{2}^{2}, \eta=\frac{\beta_{2}}{4}$, such that

$$
\Phi^{2}+2 \beta_{2} \Phi \Psi_{y}+\beta_{1} \Psi_{y}^{2} \geq C_{\beta_{2}}\left(\Phi^{2}+\Psi_{y}^{2}\right)
$$

and then choose $\beta_{2}$ satisfying

$$
c_{1} \beta_{1}+c_{2} \beta_{2} \leq \frac{1}{2} \underline{c} \text { and } \beta_{2} \leq \min \left\{-\frac{\overline{p_{v}}}{4 c_{1}}, \underline{c}\right\} .
$$

Finally, we choose $\delta,(\gamma-1) \mu$ and $\varepsilon$ so small that

$$
c \delta\left(\beta_{2}+1\right) \leq \frac{\beta_{1}}{2 \bar{v}^{\varepsilon}} \text { and } \bar{c}\left(\eta^{-1}+\beta_{2}+1\right)(\gamma-1) \mu+c \varepsilon^{\nu} \leq \frac{1}{2} \underline{c} .
$$

With these constants at hand, it follows from Gronwall type inequality that

$$
\begin{aligned}
& \left\|\left(\Phi, \Psi, \frac{W}{\sqrt{\gamma-1}}\right)(\cdot, \tau)\right\|_{1}^{2}+\int_{0}^{\tau}\left(\left\|\Phi_{y}(\cdot, \tau)\right\|^{2}+\left\|\left(\Psi_{y}, W_{y}\right)\right\|_{1}^{2}\right) d \tau \\
& +\int_{0}^{\tau} \int m V_{y}\left(\Psi^{2}+\frac{W^{2}}{\gamma-1}\right) d y d \tau \leq c \varepsilon^{(2 n+1) \nu-4} .
\end{aligned}
$$

This completes the proof of Lemma 3.2.

To finish the proof of Proposition 3.1, we need to establish the estimates on the higher derivatives of $(\Phi, \Psi, W)$. This is given by the following Lemma.

Lemma 3.3. Suppose the conditions in Proposition 3.1 are satisfied. Then

$$
\left\|\left(\partial_{y}^{2} \Phi, \partial_{y}^{2} \Psi, \frac{\partial_{y}^{2} W}{\gamma-1}\right)(\cdot, \tau)\right\|_{1}^{2}+\int_{0}^{\tau}\left(\left\|\partial_{y}^{2} \Phi(\cdot, \tau)\right\|_{1}^{2}+\left\|\left(\partial_{y}^{3} \Psi, \partial_{y}^{3} W\right)\right\|_{1}^{2}\right) d \tau \leq c \varepsilon^{(2 n+1) \nu-4}
$$

with some constant $c$ independent of $\tau_{0}$ and $\varepsilon$.

Proof. Step 1 First we rewrite $(3.7)_{2}$ as

$$
\Psi_{\tau}-\dot{s}(\varepsilon \tau) \Psi_{y}+\chi_{1} \Phi_{y}+\overline{p_{\theta}}\left\{W_{y}+\frac{\gamma-1}{R}\left(\overline{u_{y}^{\varepsilon}} \Psi-\frac{1}{2} \Psi_{y}^{2}\right)\right\}=\frac{\Psi_{y y}}{v^{\varepsilon}}-Q_{1}+q_{5}-q_{6},
$$

where $\chi_{1}=\overline{p_{v}}+\frac{\overline{u_{y}^{\varepsilon}}}{\bar{v}^{\varepsilon} v^{\varepsilon}}$. Applying $\partial_{y}^{l}$ to (3.22), multiplying both sides of the resulting equation by $\partial_{y}^{l+1} \Phi$, and integrating over $R^{1} \times[0, \tau]$, we obtain

$$
\begin{aligned}
& \int_{0}^{\tau} \int\left(\partial_{y}^{l+1} \Phi \partial_{y}^{l} \Psi_{\tau}-\dot{s} \partial_{y}^{l+1} \Phi \partial_{y}^{l+1} \Psi\right) d y d \tau \\
& +\int_{0}^{\tau} \int \partial_{y}^{l+1} \Phi \partial_{y}^{l}\left\{\chi_{1} \Phi_{y}+\bar{p}_{\theta}\left(W_{y}+\frac{\gamma-1}{R}\left(\overline{u_{y}^{\varepsilon}} \Psi-\frac{1}{2} \Psi_{y}^{2}\right)\right)\right\} d y d \tau
\end{aligned}
$$




$$
=\int_{0}^{\tau} \int \partial_{y}^{l+1} \Phi \partial_{y}^{l}\left(\frac{\Psi_{y y}}{v^{\varepsilon}}\right) d y d \tau-\int_{0}^{\tau} \int \partial_{y}^{l+1} \Phi \partial_{y}^{l}\left(Q_{1}-q_{5}+q_{6}\right) d y d \tau .
$$

Similar to the methods we have used, with the help of $(3.7)_{1}$ and $(2.49)_{1}$, we have

$$
\begin{aligned}
\int_{0}^{\tau} \int \partial_{y}^{l+1} \Phi \partial_{y}^{l} \Psi_{\tau} d y d \tau= & \int \partial_{y}^{l+1} \Phi \partial_{y}^{l} \Psi d y+\int_{0}^{\tau} \int\left(\partial_{y}^{l+1} \Psi\right)^{2} d y d \tau \\
& +\int_{0}^{\tau} \int \dot{s} \partial_{y}^{l+1} \Phi \partial_{y}^{l+1} \Psi d y d \tau
\end{aligned}
$$

and

$$
\begin{aligned}
& \int_{0}^{\tau} \iint \partial_{y}^{l+1} \Phi \partial_{y}^{l}\left(\frac{\Psi_{y y}}{v^{\varepsilon}}\right) d y d \tau \\
& =\int_{0}^{\tau} \int \frac{1}{v^{\varepsilon}} \partial_{y}^{l+1} \Phi \partial_{y}^{l+2} \Psi d y d \tau+\int_{0}^{\tau} \int \partial_{y}^{l+1} \Phi\left[\partial_{y}^{l}, \frac{1}{v^{\varepsilon}}\right] \Psi_{y y} d y d \tau \\
& =\int_{0}^{\tau} \int \frac{1}{v^{\varepsilon}} \partial_{y}^{l+1} \Phi\left(\partial_{y}^{l+1} \Phi_{\tau}-\dot{s} \partial_{y}^{l+2} \Phi\right) d y d \tau+\int_{0}^{\tau} \int \partial_{y}^{l+1} \Phi\left[\partial_{y}^{l}, \frac{1}{v^{\varepsilon}}\right] \Psi_{y y} d y d \tau \\
& =\frac{1}{2} \int \frac{\left(\partial_{y}^{l+1} \Phi\right)^{2}}{v^{\varepsilon}} d y+\frac{1}{2} \int_{0}^{\tau} \int \frac{v_{\tau}^{\varepsilon}-\dot{s} v_{y}^{\varepsilon}}{\left(v^{\varepsilon}\right)^{2}}\left(\partial_{y}^{l+1} \Phi\right)^{2} d y d \tau \\
& +\int_{0}^{\tau} \int \partial_{y}^{l+1} \Phi\left[\partial_{y}^{l}, \frac{1}{v^{\varepsilon}}\right] \Psi_{y y} d y d \tau \\
& =\frac{1}{2} \int \frac{\left(\partial_{y}^{l+1} \Phi\right)^{2}}{v^{\varepsilon}} d y+\frac{1}{2} \int_{0}^{\tau} \int \frac{\Psi_{y y}}{\left(v^{\varepsilon}\right)^{2}}\left(\partial_{y}^{l+1} \Phi\right)^{2} d y d \tau+\frac{1}{2} \int_{0}^{\tau} \int \frac{\bar{u}_{y}^{\varepsilon}}{\left(v^{\varepsilon}\right)^{2}}\left(\partial_{y}^{l+1} \Phi\right)^{2} d y d \tau \\
& +\int_{0}^{\tau} \int \partial_{y}^{l+1} \Phi\left[\partial_{y}^{l}, \frac{1}{v^{\varepsilon}}\right] \Psi_{y y} d y d \tau
\end{aligned}
$$

where $[\cdot, \cdot] \cdot$ denotes the commutator. Insert them into (3.23) to get

$$
\begin{aligned}
\frac{1}{2} \int \frac{\left(\partial_{y}^{l+1} \Phi\right)^{2}}{v^{\varepsilon}} d y= & \int \partial_{y}^{l+1} \Phi \partial_{y}^{l} \Psi d y+\int_{0}^{\tau} \int\left(\partial_{y}^{l+1} \Psi\right)^{2} d y d \tau \\
& +\int_{0}^{\tau} \int \partial_{y}^{l+1} \Phi \partial_{y}^{l}\left(\chi_{1} \Phi_{y}\right) d y d \tau-\frac{1}{2} \int_{0}^{\tau} \int \frac{\overline{u_{y}^{\varepsilon}}}{\left(v^{\varepsilon}\right)^{2}}\left(\partial_{y}^{l+1} \Phi\right)^{2} d y d \tau \\
& -\frac{1}{2} \int_{0}^{\tau} \int \frac{\Psi_{y y}}{\left(v^{\varepsilon}\right)^{2}}\left(\partial_{y}^{l+1} \Phi\right)^{2} d y d \tau-\int_{0}^{\tau} \int \partial_{y}^{l+1} \Phi\left[\partial_{y}^{l}, \frac{1}{v^{\varepsilon}}\right] \Psi_{y y} d y d \tau \\
& +\int_{0}^{\tau} \int \partial_{y}^{l+1} \Phi \partial_{y}^{l}\left\{\overline{p_{\theta}}\left(W_{y}+\frac{\gamma-1}{R}\left(\overline{u_{y}^{\varepsilon}} \Psi-\frac{1}{2} \Psi_{y}^{2}\right)\right)\right\} d y d \tau \\
& +\int_{0}^{\tau} \int \partial_{y}^{l+1} \Phi \partial_{y}^{l}\left(Q_{1}-q_{5}+q_{6}\right) d y d \tau .
\end{aligned}
$$

In the case $l=1,(3.24)$ reads

$$
\begin{aligned}
\frac{1}{2} \int \frac{\Phi_{y y}^{2}}{v^{\varepsilon}} d y= & \int \Phi_{y y} \Psi_{y} d y+\int_{0}^{\tau} \int \Psi_{y y}^{2} d y d \tau \\
& +\int_{0}^{\tau} \int\left(\chi_{1}-\frac{u_{y}^{\varepsilon}}{2\left(v^{\varepsilon}\right)^{2}}\right) \Phi_{y y}^{2} d y d \tau+\int_{0}^{\tau} \int \chi_{1 y} \Phi_{y y} \Phi_{y} d y d \tau \\
& +\frac{1}{2} \int_{0}^{\tau} \int \frac{1}{\left(v^{\varepsilon}\right)^{2}} \Psi_{y y} \Phi_{y y}^{2} d y d \tau+\int_{0}^{\tau} \int \frac{v_{y}^{\varepsilon}}{\left(v^{\varepsilon}\right)^{2}} \Phi_{y y} \Psi_{y y} d y d \tau
\end{aligned}
$$




$$
\begin{aligned}
& +\int_{0}^{\tau} \int \Phi_{y y}\left\{\bar{p}_{\theta}\left(W_{y}+\frac{\gamma-1}{R}\left(\overline{u_{y}^{\varepsilon}} \Psi-\frac{1}{2} \Psi_{y}^{2}\right)\right)\right\}_{y} d y d \tau \\
& +\int \Phi_{y y}\left(Q_{1 y}-q_{5 y}+q_{6 y}\right) d y d \tau
\end{aligned}
$$

Next we use the result we have obtained in Lemma 3.2 to estimate each term on the right hand side of (3.25). First, by Young's inequality,

$$
\int \Phi_{y y} \Psi_{y} d y \leq \frac{1}{4} \int \frac{\Phi_{y y}^{2}}{v^{\varepsilon}} d y+c\left\|\Psi_{y}\right\|^{2} \leq \frac{1}{4} \int \frac{\Phi_{y y}^{2}}{v^{\varepsilon}} d y+c \varepsilon^{(2 n+1) \nu-4} .
$$

By the definition of $\chi_{1}$, we have

$$
\chi_{1 y}=O(1)\left(1+\Phi_{y y}\right), \quad \chi_{1 y y}=O(1)\left(1+\Phi_{y y}+\Phi_{y y}^{2}+\Phi_{y y y}\right) .
$$

It follows from this and the facts $\overline{u_{y}^{\varepsilon}}=m U_{y}+O(1) \varepsilon$ and $U_{y}<0$ that

$$
\begin{aligned}
& \int_{0}^{\tau} \int\left(\chi_{1}-\frac{\overline{u_{y}^{\varepsilon}}}{2\left(v^{\varepsilon}\right)^{2}}\right) \Phi_{y y}^{2} d y d \tau \\
& =\int_{0}^{\tau} \int \overline{p_{v}} \Phi_{y y}^{2} d y d \tau+\int_{0}^{\tau} \int\left(\frac{\overline{u_{y}^{\varepsilon}} \Phi_{y}}{\overline{v^{\varepsilon}}\left(v^{\varepsilon}\right)^{2}}+\frac{\overline{u_{y}^{\varepsilon}}}{2\left(v^{\varepsilon}\right)^{2}}\right) \Phi_{y y}^{2} d y d \tau \\
& \leq \int_{0}^{\tau} \int \overline{p_{v}} \Phi_{y y}^{2} d y d \tau+c(\delta+\varepsilon) \int_{0}^{\tau}\left\|\Phi_{y y}\right\|^{2} d \tau,
\end{aligned}
$$

and

$$
\begin{aligned}
\int_{0}^{\tau} \int \chi_{1 y} \Phi_{y} \Phi_{y y} d y d \tau & \leq c \delta \int_{0}^{\tau}\left\|\Phi_{y y}\right\|^{2} d \tau-\frac{1}{8} \int_{0}^{\tau} \int \overline{p_{v}} \Phi_{y y}^{2} d y d \tau+c \int_{0}^{\tau}\left\|\Phi_{y}\right\|^{2} d \tau \\
& \leq c \delta \int_{0}^{\tau}\left\|\Phi_{y y}\right\|^{2} d \tau-\frac{1}{8} \int_{0}^{\tau} \int \overline{p_{v}} \Phi_{y y}^{2} d y d \tau+c \varepsilon^{(2 n+1) \nu-4}
\end{aligned}
$$

By Sobolev's inequality and Young's inequality, the remaining terms on the right are estimated as follows.

$$
\frac{1}{2} \int_{0}^{\tau} \int \frac{1}{\left(v^{\varepsilon}\right)^{2}} \Psi_{y y} \Phi_{y y}^{2} d y d \tau \leq c \delta \int_{0}^{\tau}\left\|\Phi_{y y}\right\|^{2} d \tau
$$

and

$$
\begin{aligned}
\int_{0}^{\tau} \int \frac{\overline{v_{y}^{\varepsilon}}}{\left(v^{\varepsilon}\right)^{2}} \Phi_{y y} \Psi_{y y} d y d \tau & \leq-\frac{1}{8} \int_{0}^{\tau} \int \overline{p_{v}} \Phi_{y y}^{2} d y d \tau+c \int_{0}^{\tau}\left\|\Psi_{y y}\right\|^{2} d \tau \\
& \leq-\frac{1}{8} \int_{0}^{\tau} \int \overline{p_{v}} \Phi_{y y}^{2} d y d \tau+c \varepsilon^{(2 n+1) \nu-4} .
\end{aligned}
$$

Continuing, using Lemma 2.3 and Lemma 3.2, we obtain

$$
\begin{aligned}
& \int_{0}^{\tau} \int \Phi_{y y}\left\{\bar{p}_{\theta}\left(W_{y}+\frac{\gamma-1}{R}\left(\overline{u_{y}^{\varepsilon}} \Psi-\frac{1}{2} \Psi_{y}^{2}\right)\right)\right\}_{y} d y d \tau \\
& \leq-\frac{1}{8} \int_{0}^{\tau} \int \overline{p_{v}} \Phi_{y y}^{2} d y d \tau \\
& \quad+c \int_{0}^{\tau} \int\left(W_{y}^{2}+W_{y y}^{2}+\left(\bar{u}_{y}^{\varepsilon^{2}}+u_{y y}^{\bar{\varepsilon}}{ }^{2}\right) \Psi^{2}+\Psi_{y}^{2}+\Psi_{y}^{4}+\Psi_{y}^{2} \Psi_{y y}^{2}\right) d y d \tau
\end{aligned}
$$




$$
\begin{aligned}
\leq & -\frac{1}{8} \int_{0}^{\tau} \int \overline{p_{v}} \Phi_{y y}^{2} d y d \tau+c \int_{0}^{\tau}\left(\left\|\Psi_{y}\right\|^{2}+\left\|\Psi_{y}\right\|_{L^{\infty}}^{2}\left\|\Psi_{y}\right\|_{1}^{2}+\left\|W_{y}\right\|_{1}^{2}\right) d \tau \\
& +c \int_{0}^{\tau} \int m V_{y} \Psi^{2} d y d \tau+c \varepsilon \tau \sup _{0 \leq \tau \leq \tau_{0}}\|\Psi(\cdot, \tau)\|^{2} \\
\leq & -\frac{1}{8} \int_{0}^{\tau} \int \overline{p_{v}} \Phi_{y y}^{2} d y d \tau+c \varepsilon^{(2 n+1) \nu-4}
\end{aligned}
$$

provided that $\left\|\Psi_{y}\right\|_{L^{\infty}}$ is bounded. By the definition of $Q_{1}$, we have

$$
\begin{aligned}
\int_{0}^{\tau} \int Q_{1 y}^{2} d y d \tau \leq & c \int_{0}^{\tau} \int\left\{\Phi_{y}^{4}+W_{y}^{4}+\bar{u}_{y}^{\varepsilon^{4}} \Psi^{4}+\Psi_{y}^{8}+\left(\Phi_{y}^{2}+W_{y}^{2}+\bar{u}_{y}^{\varepsilon^{2}} \Psi^{2}\right.\right. \\
& \left.\left.+\Psi_{y}^{4}\right)\left(\Phi_{y y}^{2}+W_{y y}^{2}+\bar{u}_{y y}^{\bar{\varepsilon}^{2}} \Psi^{2}+\Psi_{y}^{2}+\Psi_{y}^{2} \Psi_{y y}^{2}\right)\right\} d y d \tau \\
\leq & c \delta \int_{0}^{\tau} \int \Phi_{y y}^{2} d y d \tau+c \int_{0}^{\tau}\left(\left\|\Phi_{y}\right\|^{2}+\left\|\Psi_{y}\right\|_{1}^{2}+\left\|W_{y}\right\|_{1}^{2}\right) d \tau \\
& +c \int_{0}^{\tau} \int m V_{y} \Psi^{2} d y d \tau+c \varepsilon \tau \sup _{0 \leq \tau \leq \tau_{0}}\|\Psi(\cdot, \tau)\|^{2} \\
\leq & c \delta \int_{0}^{\tau} \int \Phi_{y y}^{2} d y d \tau+c \varepsilon^{(2 n+1) \nu-4}
\end{aligned}
$$

provided that $\left\|\left(\Phi_{y}, \Psi, \Psi_{y}, W_{y}\right)\right\|_{L^{\infty}}$ is bounded. Then combining Lemma 2.2, one finds

$$
\begin{aligned}
& \int \Phi_{y y}\left(Q_{1 y}-q_{5 y}+q_{6 y}\right) d y d \tau \\
\leq & -\frac{1}{8} \int_{0}^{\tau} \int \overline{p_{v}} \Phi_{y y}^{2} d y d \tau+c \int_{0}^{\tau} \int\left(Q_{1 y}^{2}+q_{5 y}^{2}+q_{6 y}^{2}\right) d y d \tau \\
\leq & -\frac{1}{8} \int_{0}^{\tau} \int \overline{p_{v}} \Phi_{y y}^{2} d y d \tau+c \delta \int_{0}^{\tau} \int \Phi_{y y}^{2} d y d \tau+c \varepsilon^{(2 n+1) \nu-4} .
\end{aligned}
$$

Collecting all the estimates we have obtained and taking $\delta$ and $\varepsilon$ to be sufficiently small, we get

$$
\int \frac{\Phi_{y y}^{2}}{v^{\varepsilon}}-\int_{0}^{\tau} \int \overline{p_{v}} \Phi_{y y}^{2} d y d \tau \leq c \varepsilon^{(2 n+1) \nu-4} .
$$

This implies

$$
\left\|\Phi_{y y}(\cdot, \tau)\right\|^{2}+\int_{0}^{\tau}\left\|\Phi_{y y}(\cdot, \tau)\right\|^{2} d \tau \leq c \varepsilon^{(2 n+1) \nu-4} .
$$

Step 2 We rewrite (3.17) as

$$
\left\{\begin{array}{l}
\Phi_{\tau}-\dot{s}(\varepsilon \tau) \Phi_{y}-\Psi_{y}=0, \\
\Psi_{\tau}-\dot{s}(\varepsilon \tau) \Psi_{y}+p\left(v^{\varepsilon}, \theta^{\varepsilon}\right)-p\left(\overline{v^{\varepsilon}}, \overline{\theta^{\varepsilon}}\right)=\frac{\Psi_{y y}}{v^{\varepsilon}}-\frac{\overline{u_{y}^{\varepsilon}}}{\overline{v^{\varepsilon}} v^{\varepsilon}} \Phi_{y}+q_{5}-q_{6}, \\
\frac{R}{\gamma-1}\left(W_{\tau}-\dot{s}(\varepsilon \tau) W_{y}\right)+\left(\overline{u_{\tau}^{\varepsilon}}-\dot{s}(\varepsilon \tau) \overline{u_{y}^{\varepsilon}}\right) \Psi+\chi_{2} \Psi_{y} \\
=\frac{W_{y y}}{v^{\varepsilon}}+\frac{\gamma-1}{R v^{\varepsilon}}\left(\bar{u}_{y}^{\varepsilon} \Psi-\frac{1}{2} \Psi_{y}^{2}\right)_{y}-\frac{\bar{\theta}_{y}^{\varepsilon}}{\bar{v}^{\varepsilon} v^{\varepsilon}} \Phi_{y}+\frac{1}{v^{\varepsilon}} \Psi_{y} \Psi_{y y} \\
-\bar{u}^{\varepsilon}\left(q_{5}-q_{6}\right)+q_{11}-q_{12}, \\
\Phi(y, 0)=\Psi(y, 0)=W(y, 0)=0,
\end{array}\right.
$$


where $\chi_{2}=p\left(v^{\varepsilon}, \theta^{\varepsilon}\right)-\frac{\bar{u}_{y}^{\varepsilon}}{v^{\varepsilon}}$. Applying $\partial_{y}^{k}$ to $(3.29)_{2}$, multiplying both sides of the resulting equation by $\partial_{y}^{k} \Psi$ and integrating on $R^{1} \times[0, T]$, we obtain

$$
\begin{aligned}
& \frac{1}{2}\left\|\partial_{y}^{k} \Psi(\cdot, \tau)\right\|^{2}+\int_{0}^{\tau} \int \frac{\left(\partial_{y}^{k+1} \Psi\right)^{2}}{v^{\varepsilon}} d y d \tau=\int_{0}^{\tau} \int \partial_{y}^{k+1} \Psi \partial_{y}^{k-1}\left(\frac{\bar{u}_{y}^{\varepsilon}}{\bar{v}^{\varepsilon} v^{\varepsilon}} \Phi_{y}\right) d y d \tau \\
& \quad+\int_{0}^{\tau} \int \partial_{y}^{k+1} \Psi \partial_{y}^{k-1}\left\{p\left(v^{\varepsilon}, \theta^{\varepsilon}\right)-p\left(\bar{v}^{\varepsilon}, \overline{\theta^{\varepsilon}}\right)\right\} d y d \tau \\
& \quad-\int_{0}^{\tau} \int \partial_{y}^{k+1} \Psi\left[\partial_{y}^{k-1}, \frac{1}{v^{\varepsilon}}\right] \Psi_{y y} d y d \tau-\int_{0}^{\tau} \int \partial_{y}^{k+1} \Psi \partial_{y}^{k-1}\left(q_{5}-q_{6}\right) d y d \tau .
\end{aligned}
$$

In the case $k=2$, by the Young's inequality and Sobolev's inequality, we have

$$
\begin{aligned}
& \frac{1}{2}\left\|\partial_{y}^{2} \Psi(\cdot, \tau)\right\|^{2}+\int_{0}^{\tau} \int \frac{\left(\partial_{y}^{3} \Psi\right)^{2}}{v^{\varepsilon}} d y d \tau \\
= & \int_{0}^{\tau} \int \partial_{y}^{3} \Psi\left(\frac{\overline{u_{y}^{\varepsilon}}}{\bar{v}^{\varepsilon} v^{\varepsilon}} \Phi_{y y}+\left(\frac{\overline{u_{y}^{\varepsilon}}}{\bar{v}^{\varepsilon} v^{\varepsilon}}\right)_{y} \Phi_{y}\right) d y d \tau+\int_{0}^{\tau} \int \partial_{y}^{3} \Psi\left\{p\left(v^{\varepsilon}, \theta^{\varepsilon}\right)-p\left(\overline{v^{\varepsilon}}, \overline{\theta^{\varepsilon}}\right)\right\}_{y} d y d \tau \\
& -\int_{0}^{\tau} \int\left(\frac{1}{v^{\varepsilon}}\right)_{y} \partial_{y}^{3} \Psi \partial_{y}^{2} \Psi d y d \tau-\int_{0}^{\tau} \int \partial_{y}^{3} \Psi\left(q_{5 y}-q_{6 y}\right) d y d \tau \\
\leq & \frac{1}{2} \int_{0}^{\tau} \int \frac{\left(\partial_{y}^{3} \Psi\right)^{2}}{v^{\varepsilon}} d y d \tau+c \int_{0}^{\tau}\left(\left\|\Phi_{y}\right\|_{1}^{2}+\left\|\Psi_{y}\right\|_{1}^{2}+\left\|W_{y}\right\|_{1}^{2}\right) d \tau \\
& +c \int_{0}^{\tau} \int m V_{y} \Psi^{2} d y d \tau+c \varepsilon \tau \sup _{0 \leq \tau \leq \tau_{0}}\|\Psi(\cdot, \tau)\|^{2}+\int_{0}^{\tau} \int\left(q_{5 y}^{2}+q_{6 y}^{2}\right) d y d \tau \\
\leq & \frac{1}{2} \int_{0}^{\tau} \int \frac{\left(\partial_{y}^{3} \Psi\right)^{2}}{v^{\varepsilon}} d y d \tau+c \varepsilon^{(2 n+1) \nu-4},
\end{aligned}
$$

provided that $\|\Phi\|_{2},\|\Psi\|_{3}$ and $\|W\|_{2}$ are bounded, where we have used (3.11) and (3.28). This implies

$$
\left\|\partial_{y}^{2} \Psi(\cdot, \tau)\right\|^{2}+\int_{0}^{\tau}\left\|\partial_{y}^{3} \Psi(\cdot, \tau)\right\|^{2} d \tau \leq c \varepsilon^{(2 n+1) \nu-4} .
$$

Applying $\partial_{y}^{k}$ to $(3.29)_{3}$, multiplying both sides of the resulting equation by $\partial_{y}^{k} W$ and integrating on $R^{1} \times[0, T]$, we get

$$
\begin{aligned}
& \frac{R}{2(\gamma-1)}\left\|\partial_{y}^{k} W(\cdot, \tau)\right\|^{2}+\int_{0}^{\tau} \int \frac{\left(\partial_{y}^{k+1} W\right)^{2}}{v^{\varepsilon}} d y d \tau \\
& =\int_{0}^{\tau} \int \partial_{y}^{k+1} W \partial_{y}^{k-1}\left\{\left(\bar{u}_{\tau}^{\varepsilon}-\dot{s}(\varepsilon \tau) \overline{u_{y}^{\varepsilon}}\right) \Psi+\chi_{2} \Psi_{y}\right\} d y d \tau \\
& \quad-\int_{0}^{\tau} \int \partial_{y}^{k+1} W\left[\partial_{y}^{k-1}, \frac{1}{v^{\varepsilon}}\right] W_{y y} d y d \tau \\
& \quad+\int_{0}^{\tau} \int \partial_{y}^{k+1} W \partial_{y}^{k-1}\left\{\frac{\bar{\theta}_{y}^{\varepsilon}}{\bar{v}^{\varepsilon} v^{\varepsilon}} \Phi_{y}-\frac{\gamma-1}{R v^{\varepsilon}}\left(\bar{u}_{y}^{\bar{\varepsilon}} \Psi-\frac{1}{2} \Psi_{y}^{2}\right)_{y}-\frac{1}{v^{\varepsilon}} \Psi_{y} \Psi_{y y}\right\} d y d \tau \\
& \quad+\int_{0}^{\tau} \int \partial_{y}^{k+1} W \partial_{y}^{k-1}\left\{\bar{u}^{\varepsilon}\left(q_{5}-q_{6}\right)-\left(q_{11}-q_{12}\right)\right\} d y d \tau .
\end{aligned}
$$

In the case $k=2$, one has

$$
\frac{R}{2(\gamma-1)}\left\|\partial_{y}^{2} W(\cdot, \tau)\right\|^{2}+\int_{0}^{\tau} \int \frac{\left(\partial_{y}^{3} W\right)^{2}}{v^{\varepsilon}} d y d \tau
$$




$$
\begin{aligned}
= & \int_{0}^{\tau} \int \partial_{y}^{3} W\left\{\left(\overline{u_{\tau}^{\varepsilon}}-\dot{s}(\varepsilon \tau) \overline{u_{y}^{\varepsilon}}\right) \Psi+\chi_{2} \Psi_{y}\right\}_{y} d y d \tau-\int_{0}^{\tau} \int\left(\frac{1}{v^{\varepsilon}}\right)_{y} \partial_{y}^{3} W \partial_{y}^{2} W d y d \tau \\
& +\int_{0}^{\tau} \int \partial_{y}^{3} W\left\{\frac{\bar{\theta}_{y}^{\varepsilon}}{\bar{v}^{\varepsilon} v^{\varepsilon}} \Phi_{y}-\frac{\gamma-1}{R v^{\varepsilon}}\left(\bar{u}_{y}^{\varepsilon} \Psi-\frac{1}{2} \Psi_{y}^{2}\right)_{y}-\frac{1}{v^{\varepsilon}} \Psi_{y} \Psi_{y y}\right\}_{y} d y d \tau \\
& +\int_{0}^{\tau} \int \partial_{y}^{3} W\left\{\left(\bar{u}^{\varepsilon}\left(q_{5}-q_{6}\right)_{y}-\left(q_{11 y}-q_{12 y}\right)\right\} d y d \tau\right. \\
\leq & \frac{1}{2} \int_{0}^{\tau} \int \frac{\left(\partial_{y}^{3} W\right)^{2}}{v^{\varepsilon}} d y d \tau+c \int_{0}^{\tau}\left(\left\|\Phi_{y}\right\|_{1}^{2}+\left\|\Psi_{y}\right\|_{2}^{2}+\left\|W_{y}\right\|_{1}^{2}\right) d \tau \\
& +c \int_{0}^{\tau} \int m V_{y} \Psi^{2} d y d \tau+c \varepsilon \tau \sup _{0 \leq \tau \leq \tau_{0}}\|\Psi(\cdot, \tau)\|^{2} \\
& +\int_{0}^{\tau} \int\left(q_{5}^{2}+q_{5 y}^{2}+q_{6}^{2}+q_{6 y}^{2}+q_{11 y}^{2}+q_{12 y}^{2}\right) d y d \tau \\
\leq & \frac{1}{2} \int_{0}^{\tau} \int \frac{\left(\partial_{y}^{3} W\right)^{2}}{v^{\varepsilon}} d y d \tau+c \varepsilon^{(2 n+1) \nu-4},
\end{aligned}
$$

provided that $\|\Phi\|_{2},\|\Psi\|_{3}$ and $\|W\|_{3}$ are bounded, where we have used (3.11), (3.28), (3.31) and Lemma 2.2-2.3. This gives

$$
\left\|\frac{\partial_{y}^{2} W}{\sqrt{\gamma-1}}(\cdot, \tau)\right\|^{2}+\int_{0}^{\tau}\left\|\partial_{y}^{3} W(\cdot, \tau)\right\|^{2} d \tau \leq c \varepsilon^{(2 n+1) \nu-4}
$$

Step 3 Similar to Step 1, for $l=2$, due to (3.8), (3.11), (3.28), (3.31) and (3.33), we have

$$
\begin{aligned}
& \frac{1}{2} \int \frac{\left(\partial_{y}^{3} \Phi\right)^{2}}{v^{\varepsilon}} d y-\int_{0}^{\tau} \int \overline{p_{v}}\left(\partial_{y}^{3} \Phi\right)^{2} d y d \tau \\
& =\int \partial_{y}^{3} \Phi \partial_{y}^{2} \Psi d y+\int_{0}^{\tau} \int\left(\partial_{y}^{3} \Psi\right)^{2} d y d \tau+\int_{0}^{\tau} \int\left(\frac{\bar{u}_{y}^{\varepsilon} \Phi_{y}}{\bar{v}^{\varepsilon}\left(v^{\varepsilon}\right)^{2}}+\frac{\bar{u}_{y}^{\varepsilon}}{2\left(v^{\varepsilon}\right)^{2}}\right)\left(\partial_{y}^{3} \Phi\right)^{2} d y d \tau \\
& +\int_{0}^{\tau} \int\left(2 \chi_{1 y} \Phi_{y y}+\chi_{1 y y} \Phi_{y}\right) \partial_{y}^{3} \Phi d y d \tau+\frac{1}{2} \int_{0}^{\tau} \int \frac{\Psi_{y y}}{\left(v^{\varepsilon}\right)^{2}}\left(\partial_{y}^{3} \Phi\right)^{2} d y d \tau \\
& +\int_{0}^{\tau} \int \partial_{y}^{3} \Phi\left\{\frac{2\left(\Phi_{y y}+\bar{v}_{y}^{\varepsilon}\right)}{\left(v^{\varepsilon}\right)^{2}} \partial_{y}^{3} \Psi+\left(\frac{v_{y y}^{\bar{\varepsilon}}}{\left(v^{\varepsilon}\right)^{2}}-\frac{2\left(\Phi_{y y}+\bar{v}_{y}^{\varepsilon}\right)^{2}}{\left(v^{\varepsilon}\right)^{3}}\right) \partial_{y}^{2} \Psi\right\} d y d \tau \\
& +\int_{0}^{\tau} \int \partial_{y}^{3} \Phi \partial_{y}^{2}\left\{\overline{p_{\theta}}\left(W_{y}+\frac{\gamma-1}{R}\left(\bar{u}_{y}^{\varepsilon} \Psi-\frac{1}{2} \Psi_{y}^{2}\right)\right)\right\} d y d \tau \\
& +\int_{0}^{\tau} \int \partial_{y}^{3} \Phi\left(\partial_{y}^{2} Q_{1}-\partial_{y}^{2} q_{5}+\partial_{y}^{2} q_{6}\right) d y d \tau \\
& \leq \frac{1}{4} \int \frac{\left(\partial_{y}^{3} \Phi\right)^{2}}{v^{\varepsilon}} d y+c\left\|\partial_{y}^{2} \Psi(\cdot, \tau)\right\|^{2}+c(\delta+\varepsilon) \int_{0}^{\tau}\left\|\partial_{y}^{3} \Phi\right\|^{2} d \tau \\
& -\frac{1}{2} \int_{0}^{\tau} \int \overline{p_{v}}\left(\partial_{y}^{3} \Phi\right)^{2} d y d \tau+c \int_{0}^{\tau}\left(\left\|\Phi_{y}\right\|_{1}^{2}+\left\|\Psi_{y}\right\|_{2}^{2}+\left\|W_{y}\right\|_{2}^{2}\right) d \tau \\
& +c \int_{0}^{\tau} \int m V_{y} \Psi^{2} d y d \tau+c \varepsilon \tau \sup _{0 \leq \tau \leq \tau_{0}}\|\Psi(\cdot, \tau)\|^{2} \\
& +c \int_{0}^{\tau} \int\left\{\left(\partial_{y}^{2} Q_{1}\right)^{2}+\left(\partial_{y}^{2} q_{5}\right)^{2}+\left(\partial_{y}^{2} q_{6}\right)^{2}\right\} d y d \tau \\
& \leq \frac{1}{4} \int \frac{\left(\partial_{y}^{3} \Phi\right)^{2}}{v^{\varepsilon}} d y-\frac{1}{2} \int_{0}^{\tau} \int \overline{p_{v}}\left(\partial_{y}^{3} \Phi\right)^{2} d y d \tau+c(\delta+\varepsilon) \int_{0}^{\tau}\left\|\partial_{y}^{3} \Phi\right\|^{2} d \tau+c \varepsilon^{(2 n+1) \nu-4}
\end{aligned}
$$


provided that $\|(\Phi, \Psi, W)\|_{3}$ is bounded. By taking $\delta$ and $\varepsilon$ to be sufficiently small, we arrive at

$$
\int \frac{\left(\partial_{y}^{3} \Phi\right)^{2}}{v^{\varepsilon}} d y-\int_{0}^{\tau} \int \overline{p_{v}}\left(\partial_{y}^{3} \Phi\right)^{2} d y d \tau \leq c \varepsilon^{(2 n+1) \nu-4}
$$

which implies

$$
\left\|\partial_{y}^{3} \Phi(\cdot, \tau)\right\|^{2}+\int_{0}^{\tau}\left\|\partial_{y}^{3} \Phi(\cdot, \tau)\right\|^{2} d \tau \leq c \varepsilon^{(2 n+1) \nu-4} .
$$

Step 4 Similarly, when $k=3$, we have

$$
\begin{aligned}
& \frac{1}{2}\left\|\partial_{y}^{3} \Psi(\cdot, \tau)\right\|^{2}+\int_{0}^{\tau} \int \frac{\left(\partial_{y}^{4} \Psi\right)^{2}}{v^{\varepsilon}} d y d \tau \\
= & \int_{0}^{\tau} \int \partial_{y}^{4} \Psi \partial_{y}^{2}\left(\frac{\overline{u_{y}^{\varepsilon}}}{\bar{v}^{\varepsilon} v^{\varepsilon}} \Phi_{y}\right) d y d \tau+\int_{0}^{\tau} \int \partial_{y}^{4} \Psi \partial_{y}^{2}\left\{p\left(v^{\varepsilon}, \theta^{\varepsilon}\right)-p\left(\overline{v^{\varepsilon}}, \bar{\theta}^{\varepsilon}\right)\right\} d y d \tau \\
& -\int_{0}^{\tau} \int \partial_{y}^{4} \Psi\left\{\partial_{y}^{2}\left(\frac{1}{v^{\varepsilon}}\right) \partial_{y}^{2} \Psi+2\left(\frac{1}{v^{\varepsilon}}\right)_{y} \partial_{y}^{3} \Psi\right\} d y d \tau-\int_{0}^{\tau} \int \partial_{y}^{4} \Psi \partial_{y}^{2}\left(q_{5}-q_{6}\right) d y d \tau \\
\leq & \frac{1}{2} \int_{0}^{\tau} \int \frac{\left(\partial_{y}^{4} \Psi\right)^{2}}{v^{\varepsilon}} d y d \tau+c \int_{0}^{\tau}\left(\left\|\Phi_{y}\right\|_{2}^{2}+\left\|\Psi_{y}\right\|_{2}^{2}+\left\|W_{y}\right\|_{2}^{2}\right) d y d \tau \\
& +c \int_{0}^{\tau} \int m V_{y} \Psi^{2} d y d \tau+c \varepsilon \tau \sup _{0 \leq \tau \leq \tau_{0}}\|\Psi(\cdot, \tau)\|^{2}+\int_{0}^{\tau} \int\left(\left(\left(\partial_{y}^{2} q_{5}\right)^{2}+\left(\partial_{y}^{2} q_{6}\right)^{2}\right) d y d \tau\right. \\
\leq & \frac{1}{2} \int_{0}^{\tau} \int \frac{\left(\partial_{y}^{4} \Psi\right)^{2}}{v^{\varepsilon}} d y d \tau+c \varepsilon^{(2 n+1) \nu-4},
\end{aligned}
$$

provided that $\|(\Phi, \Psi, W)\|_{3}$ is bounded. This implies

$$
\left\|\partial_{y}^{3} \Psi(\cdot, \tau)\right\|^{2}+\int_{0}^{\tau}\left\|\partial_{y}^{4} \Psi(\cdot, \tau)\right\|^{2} d \tau \leq c \varepsilon^{(2 n+1) \nu-4} .
$$

And for $W$, we have

$$
\begin{aligned}
& \frac{R}{2(\gamma-1)}\left\|\partial_{y}^{3} W(\cdot, \tau)\right\|^{2}+\int_{0}^{\tau} \int \frac{\left(\partial_{y}^{4} W\right)^{2}}{v^{\varepsilon}} d y d \tau \\
& =\int_{0}^{\tau} \int \partial_{y}^{4} W \partial_{y}^{2}\left\{\left(\overline{u_{\tau}^{\varepsilon}}-\dot{s}(\varepsilon \tau) \overline{u_{y}^{\varepsilon}}\right) \Psi+\chi_{2} \Psi_{y}\right\} d y d \tau \\
& \quad-\int_{0}^{\tau} \int \partial_{y}^{4} W\left\{\partial_{y}^{2}\left(\frac{1}{v^{\varepsilon}}\right) \partial_{y}^{2} W+2\left(\frac{1}{v^{\varepsilon}}\right)_{y} \partial_{y}^{3} W\right\} d y d \tau \\
& \quad+\int_{0}^{\tau} \int \partial_{y}^{4} W \partial_{y}^{2}\left\{\frac{\overline{\theta_{y}^{\varepsilon}}}{\bar{v}^{\varepsilon} v^{\varepsilon}} \Phi_{y}-\frac{\gamma-1}{R v^{\varepsilon}}\left(\bar{u}_{y}^{\varepsilon} \Psi-\frac{1}{2} \Psi_{y}^{2}\right)_{y}-\frac{1}{v^{\varepsilon}} \Psi_{y} \Psi_{y y}\right\} d y d \tau \\
& \quad+\int_{0}^{\tau} \int \partial_{y}^{4} W \partial_{y}^{2}\left\{\left(\bar{u}^{\varepsilon}\left(q_{5}-q_{6}\right)\right)-\left(q_{11}-q_{12}\right)\right\} d y d \tau \\
& \leq \frac{1}{2} \int_{0}^{\tau} \int \frac{\left(\partial_{y}^{4} W\right)^{2}}{v^{\varepsilon}} d y d \tau+c \int_{0}^{\tau}\left(\left\|\Phi_{y}\right\|_{2}^{2}+\left\|\Psi_{y}\right\|_{3}^{2}+\left\|W_{y}\right\|_{2}^{2}\right) d y d \tau \\
& \quad+c \int_{0}^{\tau} \int m V_{y} \Psi^{2} d y d \tau+c \varepsilon \tau \sup _{0 \leq \tau \leq \tau_{0}}\|\Psi(\cdot, \tau)\|^{2} \\
& \quad+\int_{0}^{\tau} \int\left(q_{5}^{2}+q_{6}^{2}+q_{5 y}^{2}+q_{6 y}^{2}+q_{5 y y}^{2}+q_{6 y y}^{2}+q_{11 y y}^{2}+q_{12 y y}^{2}\right) d y d \tau
\end{aligned}
$$




$$
\leq \frac{1}{2} \int_{0}^{\tau} \int \frac{\left(\partial_{y}^{4} W\right)^{2}}{v^{\varepsilon}} d y d \tau+c \varepsilon^{(2 n+1) \nu-4},
$$

provided that $\|(\Phi, \Psi, W)\|_{3}$ is bounded. This implies

$$
\left\|\frac{\partial_{y}^{3} W}{\sqrt{\gamma-1}}(\cdot, \tau)\right\|^{2}+\int_{0}^{\tau}\left\|\partial_{y}^{4} W(\cdot, \tau)\right\|^{2} d \tau \leq c \varepsilon^{(2 n+1) \nu-4}
$$

So far we finish the proof of Lemma 3.3.

Combining Lemma 3.2 and Lemma 3.3 together, we complete the proof of Proposition 3.1 .

4. Proof of Theorem 1.2. Using the Proposition 3.1 and the standard continuous induction argument, we conclude that

Proposition 4.1. There exist positive constants $\varepsilon_{0}, \mu_{0}$ and $C$, which are independent of $\varepsilon$ such that if $0<\varepsilon<\varepsilon_{0}$ and $0 \leq(\gamma-1) \mu \leq \mu_{0}$, then the Cauchy problem (3.7) has a unique solution $(\Phi, \Psi, W) \in C^{1}\left([0, T / \varepsilon]: H^{3}\left(R^{1}\right)\right)$. Furthermore, the following inequality holds

$$
\begin{aligned}
& \sup _{0 \leq \tau \leq T / \varepsilon}\left\|\left(\Phi, \Psi, \frac{W}{\sqrt{\gamma-1}}\right)(\cdot, \tau)\right\|_{3}^{2}+\int_{0}^{T / \varepsilon}\left(\left\|\Phi_{y}(\cdot, \tau)\right\|_{2}^{2}+\left\|\left(\Psi_{y}, W_{y}\right)(\cdot, \tau)\right\|_{3}^{2}\right) d \tau \\
& \leq C \varepsilon^{(2 n+1) \nu-4}
\end{aligned}
$$

Proof of Theorem 1.2. Now we choose $\nu \in(\eta, 1) \cap\left(\frac{4 n+1}{4 n+2}, 1\right)$. In view of $(4.1)$ and Sobolev inequality, we have

$$
\begin{aligned}
& \sup _{0 \leq t \leq T}\left\|\left(v^{\varepsilon}-\bar{v}^{\varepsilon}, u^{\varepsilon}-\bar{u}^{\varepsilon}, \theta^{\varepsilon}-\bar{\theta}^{\varepsilon}\right)(\cdot, t)\right\|^{2} \\
& =\sup _{0 \leq t \leq T}\left\|\left(\bar{\Phi}_{x}, \bar{\Psi}_{x}, \bar{W}_{x}+\frac{\gamma-1}{R}\left(\overline{u_{x}^{\varepsilon}} \bar{\Psi}-\frac{1}{2} \bar{\Psi}_{x}^{2}\right)\right)(\cdot, t)\right\|^{2} \\
& =\varepsilon \sup _{0 \leq \tau \leq T / \varepsilon}\left\|\left(\Phi_{y}, \Psi_{y}, W_{y}+\frac{\gamma-1}{R}\left(\bar{u}_{y}^{\varepsilon} \Psi-\frac{1}{2} \Psi_{y}^{2}\right)\right)(\cdot, \tau)\right\|^{2} \\
& \leq c \varepsilon \sup _{0 \leq \tau \leq T / \varepsilon}\left(\left\|\Phi_{y}(\cdot, \tau)\right\|^{2}+\|\Psi(\cdot, \tau)\|^{2}+\left(1+\left\|\Psi_{y}(\cdot, \tau)\right\|^{4}\right)\left\|\Psi_{y}(\cdot, \tau)\right\|^{2}\right. \\
& \left.\quad+\left\|\Psi_{y y}(\cdot, \tau)\right\|^{2}+\left\|W_{y}(\cdot, \tau)\right\|^{2}\right) \\
& \leq c \varepsilon \sup _{0 \leq \tau \leq T / \varepsilon}\left(\left\|\Phi_{y}(\cdot, \tau)\right\|^{2}+\|\Psi(\cdot, \tau)\|_{2}^{2}+\left\|W_{y}(\cdot, \tau)\right\|^{2}\right) \\
& \leq C \varepsilon^{(2 n+1) \nu-3} \leq C \varepsilon^{2 n-\frac{5}{2}} .
\end{aligned}
$$

On the other hand, it follows from Lemma 2.3 that

$$
\sup _{0 \leq t \leq T}\left\|\left(\bar{v}^{\varepsilon}-v_{0}, \bar{u}^{\varepsilon}-u_{0}, \overline{\theta^{\varepsilon}}-\theta_{0}\right)(\cdot, t)\right\|^{2} \leq C \varepsilon^{\nu} \leq C \varepsilon^{\eta} .
$$

Consequently,

$$
\begin{aligned}
& \sup _{0 \leq t \leq T}\left\|\left(v^{\varepsilon}-v_{0}, u^{\varepsilon}-u_{0}, \theta^{\varepsilon}-\theta_{0}\right)(\cdot, t)\right\|^{2} \\
& \leq \sup _{0 \leq t \leq T}\left\|\left(v^{\varepsilon}-\bar{v}^{\varepsilon}, u^{\varepsilon}-\bar{u}^{\varepsilon}, \theta^{\varepsilon}-\overline{\theta^{\varepsilon}}\right)(\cdot, t)\right\|^{2}+\sup _{0 \leq t \leq T}\left\|\left(\bar{v}^{\varepsilon}-v_{0}, \overline{u^{\varepsilon}}-u_{0}, \overline{\theta^{\varepsilon}}-\theta_{0}\right)(\cdot, t)\right\|^{2}
\end{aligned}
$$


$\leq c \varepsilon^{\eta}$

which gives (1.11). Finally,

$$
\begin{aligned}
& \left\|\left(v^{\varepsilon}-\bar{v}^{\varepsilon}, u^{\varepsilon}-\bar{u}^{\varepsilon}, \theta^{\varepsilon}-\overline{\theta^{\varepsilon}}\right)(\cdot, t)\right\|_{L^{\infty}}=\left\|\left(\Phi_{y}, \Psi_{y}, W_{y}+\frac{\gamma-1}{R}\left(\bar{u}_{y}^{\varepsilon} \Psi-\frac{1}{2} \Psi_{y}^{2}\right)\right)(\cdot, t)\right\|_{L^{\infty}} \\
& \leq c\left\|\left(\Phi_{y}, \Psi, \Psi_{y}, W_{y}\right)(\cdot, t)\right\|^{\frac{1}{2}}\left\|\left(\Phi_{y y}, \Psi, \Psi_{y}, \Psi_{y y}, W_{y y}\right)(\cdot, t)\right\|^{\frac{1}{2}} \\
& \leq c \varepsilon^{((2 n+1) \nu-4) / 2} \leq c \varepsilon^{n-\frac{7}{4}} .
\end{aligned}
$$

This gives (1.10). By using Lemma 2.3 again, we obtain (1.12).

We completes the proof of the Theorem 1.2.

Acknowledgement. This paper is part of my Ph.D thesis. I express my hearty thanks to my supervisor, Professor Zhouping Xin, for his encouragement and guidance. I also thank Professor Tao Luo for his helpful advice on this paper.

\section{REFERENCES}

[1] J. Goodman, Nonlinear asymptotic stability of viscous shock profiles for conservation laws, Arch. Rational Mech. Anal., 95:4 (1986), pp. 325-344.

[2] J. Goodman And Z.-P. Xin, Viscous limits for piecewise smooth solutions to systems of conservation laws, Arch. Rational Mech. Anal., 121:3 (1992), pp. 235-265.

[3] H.-O. KReiss, Initial boundary value problems for hyperbolic systems, Comm. Pure Appl. Math., 23 (1970), pp. 277-298.

[4] J. RAUCH, $\mathcal{L}_{2}$ is a continuable initial condition for Kreiss' mixed problems, Comm. Pure Appl. Math., 25 (1972), pp. 265-285.

[5] S. KaWASHIma, Systems of hyperbolic-parabolic composite type, with applications to the equations of Magneto-Hydrodynamics, Doctoral Thesis, Kyoto Univ., (1983).

[6] R. J. DiPerna, Convergence of approximate solutions to conservation laws, Arch. Rational Mech. Anal., 82:1 (1983), pp. 27-70.

[7] D. Hoff And T.-P. LIU, The inviscid limit for the Navier-Stokes equations of compressible, isentropic flow with shock data, Indiana Univ. Math. J., 38:4 (1989), pp. 861-915.

[8] T.-P. LiU, Nonlinear stability of shock waves for viscous conservation laws, Mem. Amer. Math. Soc., 56 (1985), 328, v+108.

[9] Z.-P. XIN, Zero dissipation limit to rarefaction waves for the one-dimensional Navier-Stokes equations of compressible isentropic gases, Comm. Pure Appl. Math., 46:5 (1993), pp. 621665.

[10] S. JiAnG, G. Ni AND W. Sun, Vanishing viscosity limit to rarefaction waves for the NavierStokes equations of one-dimensional compressible heat-conducting fluids, SIAM J. Math. Anal., 38:2 (2006), pp. 368-384 (electronic).

[11] S.-H. YU, Zero-dissipation limit of solutions with shocks for systems of hyperbolic conservation laws, Arch. Ration. Mech. Anal., 146:4 (1999), pp. 275-370.

[12] P. C. FIFE, Dynamics of internal layers and diffusive interfaces, CBMS-NSF Regional Conference Series in Applied Mathematics, 53, Society for Industrial and Applied Mathematics (SIAM), Philadelphia, PA, 1988, vi+93.

[13] P. D. LAx, Hyperbolic systems of conservation laws and the mathematical theory of shock waves, Conference Board of the Mathematical Sciences Regional Conference Series in Applied Mathematics, No. 11, Society for Industrial and Applied Mathematics, Philadelphia, Pa., 1973, v+48.

[14] D. G. Aronson, Bounds for the fundamental solution of a parabolic equation, Bull. Amer. Math. Soc., 73 (1967), pp. 890-896.

[15] H. WANG, Viscous limits for piecewise smooth solutions of the p-system, J. Math. Anal. Appl., 299:2 (2004), pp. 411-432.

[16] S. KaWhshima And A. Matsumura, Asymptotic stability of traveling wave solutions of systems for one-dimensional gas motion, Comm. Math. Phys., 101:1 (1985), pp. 97-127.

[17] D. Gilbarg, The existence and limit behavior of the one-dimensional shock layer, Amer. J. Math., 73 (1951), pp. 256-274.

[18] S. Bianchini And A. Bressan, Vanishing viscosity solutions of nonlinear hyperbolic systems, Ann. of Math. (2), 161:1 (2005), pp. 223-342. 
S. MA 